\title{
Hydrochemistry of shallow groundwater and surface water in the Ndop plain, North West Cameroon
}

\author{
Mengnjo J. Wirmvem ${ }^{1 *}$, Takeshi Ohba ${ }^{1}$, Wilson Y. Fantong ${ }^{2}$, Samuel N. Ayonghe ${ }^{3}$, Justice Y. \\ Suila ${ }^{4}$, Asobo Nkengmatia E. Asaah ${ }^{5}$, Gregory Tanyileke ${ }^{2}$, and Joseph V. Hell ${ }^{2}$ \\ ${ }^{1}$ Department of Chemistry, School of Science, Tokai University, Hiratsuka, 259-1211, Japan. \\ ${ }^{2}$ Institute of Mining and Geological Research, P.O. Box 4110, Yaounde, Cameroon. \\ ${ }^{3}$ Department of Environmental Science, University of Buea, Box 63, Buea, Cameroon. \\ ${ }^{4}$ G.B.H.S. Atiela, Nkwen, P.O. Box 5147, Bamenda, Cameroon. \\ ${ }^{5}$ Department of Earth and Planetary Sciences, Tokyo Institute of Technology, Tokyo, 152-8551, Japan.
}

Accepted 20 May, 2013

\begin{abstract}
This study was conducted on the hydrochemistry of shallow groundwater and surface water in the Ndop plain, North West Cameroon. The objectives were to determine the physico-chemical characteristics of water, controls on water chemistry and suitability for drinking and irrigation. Forty-six shallow groundwater and 26 surface water samples were investigated. Field measurements of physical parameters were preceded by chemical analyses of the samples for major ions and $\mathrm{F}^{-}$. About $69 \%$ of the water sources had $\mathrm{pH}$ values below 6.5 , classifying the water as barely acidic. Electrical conductivity $(<282 \mu \mathrm{S} / \mathrm{cm})$ and total dissolved solids $(<183 \mathrm{mg} / \mathrm{l})$ were low suggesting low-mineralised and freshwater. The relative abundance of major ions (meq/l) was $\mathrm{Na}^{2+}>\mathrm{Ca}^{2+}>\mathrm{Mg}^{2+}>\mathrm{K}^{+}$for cations and $\mathrm{HCO}_{3}{ }^{-}$ $>\mathrm{NO}_{3} \geq \mathrm{Cl}^{-}>\mathrm{SO}_{4}{ }^{2-}$ for anions. Major ion concentrations were low, and within the WHO guidelines for drinking water, but $\mathrm{F}^{-}$concentrations were much lower $(<0.39 \mathrm{mg} / \mathrm{l})$ and below guideline value. Main water types and proportions were $\mathrm{Na}-\mathrm{HCO}_{3}(53 \%), \mathrm{Ca}-\mathrm{HCO}_{3}(35 \%)$ and a mixed $\mathrm{Na}-\mathrm{Ca}-\mathrm{HCO}_{3}(11 \%)$. Predominant processes influencing water chemistry were incongruent dissolution of silicate minerals and cation-exchange of $\mathrm{Na}^{+}$in rocks for $\mathrm{Ca}^{2+}$ in water. The low major ion concentrations indicated low water-rock interactions and short residence time. The analysed water was suitable for irrigation.
\end{abstract}

Key words: Water chemistry, hydrochemical controls, drinking-irrigation quality, Ndop plain, Cameroon.

\section{INTRODUCTION}

Groundwater and surface water geochemical studies can provide a better understanding of potential water quality variations due to geology and land use practices (Edmunds and Smedley, 1996; Deutsch, 1997; Appelo and Postma, 2005; Ramesh and Elango, 2012). In Africa, groundwater is a vital resource with nearly $80 \%$ of the continent's population relying on it as a main source of drinking water. However, in many parts of the continent, reaching basic health requirements is still a concern (Adelana and MacDonald, 2008; Adelana et al., 2011; Edet et al., 2011; Tay, 2012).
Cameroon is endowed with numerous water resources (Katte et al., 2003; Molua and Lambi, 2006), but an assessment of these resources, including their reliability, quality and sustainability has not been systematically carried out (Katte et al., 2003). Improved drinking water sanitation in semi-urban and rural communities is nearly the same from $37 \%$ in 1990 to $36 \%$ in 2010 (WHO/UNICEF, 2012). With increasing population, the high demand for water, especially in most poor rural communities, is met by shallow groundwater of generally unknown quality. This is usually the best option all year 
round as most surface water sources and springs are unreliable and run dry or reduce in size during the dry season, or become polluted by heavy floods in the rainy season.

Only few hydrochemical studies have been carried out to assess the geochemical characteristics of groundwater and its suitability for use in Cameroon (example, Njitchoua and Ngounou-Ngatcha, 1997; Katte et al., 2003; Fantong et al., 2009; Eneke et al., 2011; Ako et al., 2012). The Ndop plain is a semi-urban community where groundwater, as well as surface water, is a critical resource for human livelihood. The plain has an estimated population of over 200,000 people with ca. $70 \%$ involved in subsistence farming. Many ethnic groups have been attracted to the area as a result of fertile soils (Ndzeidze, 2008; Fonge et al., 2012). This has been followed by high demand for water and other natural resources (Fonge et al., 2012). Over $70 \%$ of the population depends solely on shallow groundwater, as well as surface water of poor microbial quality for drinking and other domestic purposes (Wirmvem et al., 2013). A hydrochemical study of water quality based on major ion composition has not been carried out. Since the development of the Bamendjin dam in the area in the 1970 s, rice cultivation is the main cash crop using the dam and river waters. Unfortunately, there is no information on the suitability of the water sources for irrigation despite the on-going programme by the Cameroon government to revamp rice cultivation in the area. The chemical composition of water and its mineralization process are imperative in classifying and assessing drinking water quality (Deutsch, 1997; WHO, 2004; Kozisek, 2005) while irrigation water quality criteria can be used as a guideline by farmers for selecting appropriate management practice to overcome potential salinity hazard (Gupta et al., 2009; Ramesh and Elango, 2012).

The present study, therefore, presents the first hydrochemical baseline dataset of groundwater and surface water in the Ndop plain. The objectives were to (1) determine the physico-chemical characteristics of the water, (2) define the factors that control major ion chemistry, and (3) evaluate their suitability for drinking and irrigation. The data will provide a hydrochemical baseline data for water quality assessment, management, and monitoring of changes in the hydrochemical system from the prevailing human activities in the area. The study will also contribute to the limited knowledge on natural water composition in North West Cameroon. With the on-going programme to revamp rice cultivation in the area, an assessment of the suitability of the water sources for irrigation is invaluable.

\section{The study area}

The study area (Figure 1) is an inter-mountain basin (ca. $1,100 \mathrm{~km}^{2}$ ) in the Western Highlands of Cameroon (WHC), along the Cameroon Volcanic Line (CVL). From the surrounding CVL rages, the relief drops steeply from an average altitude of ca. $1,899 \mathrm{~m}$ to ca. $1,200 \mathrm{~m}$ at the margins of the plain, and then gently to ca. $1,180 \mathrm{~m}$ above sea level in the south central portion. The flanks of the mountain chain are discharge points for numerous rivers and streams flowing dendritically into the plain and recharging the Bamendjin dam (Figure 1). Details on the drainage are described by Ndzeidze (2008). According to Neba (1999), the area has an Equatorial monsoon climate type characterized by high rainfall and relative humidity. This is attributed to the effect of the mountainous relief that characterises much of the WHC (Neba, 1999; Molua and Lambi, 2006). Two distinct seasons are differentiated, a long rainy season (mid-March to midNovember) and a short dry season (mid-November to mid-March). Mean annual rainfall and temperature, in 2012, were $1,540 \mathrm{~mm}$ and $26^{\circ} \mathrm{C}$, respectively (Wirmvem et al., unpublished data), but with cooler climate in adjacent high elevations (Ndzeidze, 2008; Ndenecho, 2009). The heaviest rainfall occurs from July to September and often results in floods due to the poor drainage (Ndzeidze, 2008).

Geologically, the area is a shallow Cenozoic 'sedimentary' basin, underlain mainly by a consistent Precambrian granitic basement (Figure 1). This basement forms part of the tectonically inactive African shield consisting mostly of Precambrian Basement Complex rocks (Neba, 1999; Marzoli et al., 1999). Chemical weathering of the basement and surrounding volcanic rocks has produced thick unconsolidated sediments, mainly of clay to sand sizes. The basement is largely covered with these sediments, but outcrops in certain areas. The surrounding volcanoes are represented by voluminous Q-trachytes and minor rhyolitic ignimbrites with slight to moderate alkaline basalts and minor basanites (Marzoli et al., 1999). The voluminous $Q$-trachytes are enriched in alkali feldspars $\left(\mathrm{Na}_{2} \mathrm{O}+\mathrm{K}_{2} \mathrm{O}\right)$. In-situ weathered basement and alkali-rich fluvial sediments constitute the aquifer material in the plain. From the margins of the plain towards the south-central portion, there is a progressive change from sandy to clayey sediments. The unconsolidated sandysediments form the groundwater aquifer system. This explains the concentric model of human settlement around the dam water (Ndzeidze, 2008) tapping groundwater through wells from this aquifer. The poor microbial quality of water with consequent water-borne infections (Wirmvem et al., 2013) has prompted the chlorination of water in wells by some members of the community.

\section{MATERIALS AND METHODS}

\section{Water sampling and physico-chemical measurements}

Water was collected from 72 water sources in January 2012 (dry season) within 13 communities (Figure 1). The sources comprised 32 open wells and 14 'boreholes' (hereafter termed groundwater); and 14 streams, 4 rivers, 5 dam waters, and 3 lakes (Lakes Bambili, 


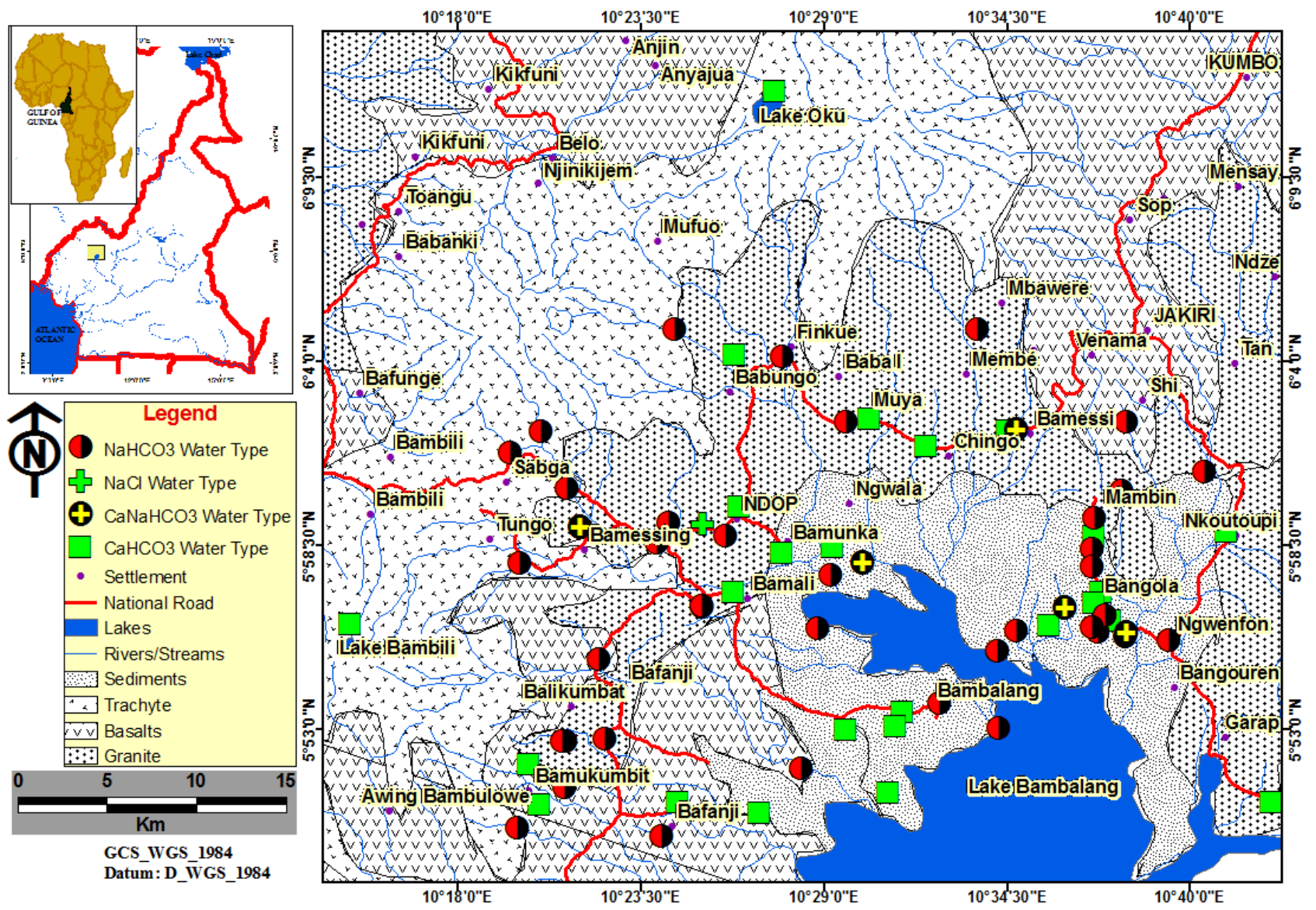

Figure 1. The geology and drainage map of Ndop Plain (modified after Ndzeidze, 2008) showing sample points/spatial distribution of water types.

Oku, and Ber) (hereafter called surface water). The sample location points (Figure 1) and altitudes were noted in the field by using a Garmin Vista CX GPS. Four sporadic rainfall events in the plain and its environs were also sampled. Field measurements and water sampling followed methods described by Karklins (1996). New 100 $\mathrm{ml}$ narrow mouth and low-density polyethylene (LDPE) bottles were properly rinsed with distilled water and dried in the Laboratory (in order to avoid contamination) and used for sampling the water. At each site, water was collected into a plastic bucket after thorough rinsing with water to be sampled. Groundwater from active wells was collected using a rope tied to a bucket and/or permanently installed hand-pumps (after several minutes of pumping). Surface water was sampled as far as possible from the edges of the water bodies and as deep as possible along the flow path. The water collected was then filtered directly into 2 LDPE bottles using a plastic syringe, fitted with a $0.20 \mu \mathrm{m}$ filter after thorough rinsing with the same water that was sampled. Collected samples were properly capped and preserved in a cooler container containing ice blocks. Water for major ion analyses was put in one bottle and water for alkalinity determination in a separate bottle. The following physical parameters: activity of hydrogen ion $(\mathrm{pH})$, electrical conductivity (EC), total dissolved solids (TDS), and water temperature were measured immediately from the remaining collected water using a multipurpose HI991300 hand held pH/EC/TDS/C Hanna Meter. The meter was calibrated before and during the field campaign using buffer solutions recommended by the manufacturer. The depths to standing water level and total depth of water column were determined with a water level indicator. Alkalinity/bicarbonate $\left(\mathrm{HCO}_{3}{ }^{-}\right)$ was determined within 8 hours of sampling by acid titration from a volume of $0.02 \mathrm{M} \mathrm{HCl}$, added to the sample and stirred with an automatic stirrer until the end-point, marked by a $\mathrm{pH}$ of 4.5 .

\section{Chemical analyses}

Chemical analyses were carried out at Tokai University, Japan within two weeks of sample collection. Major cations: $\mathrm{Na}^{+}, \mathrm{K}^{+}, \mathrm{Mg}^{2+}$ and $\mathrm{Ca}^{2+}$ were determined by a Flame Technique in a High Resolution Continuum Source AAS (ContrAA 700) as described by Welz et al. (2006). Samples with EC values $>100 \mu \mathrm{S} / \mathrm{cm}$ were diluted 5 or 10 times to acquire an absorbance within the range of the used standards for $\mathrm{Na}^{+}, \mathrm{K}^{+}$and $\mathrm{Ca}^{2+}$. The anions: $\mathrm{F}^{-}, \mathrm{Cl}^{-}, \mathrm{NO}_{3}{ }^{-}, \mathrm{SO}_{4}{ }^{2-}$, $\mathrm{PO}_{4}{ }^{3-}, \mathrm{NO}_{2}^{-}$and $\mathrm{Br}^{-}$were determined by using an lon Chromatography (Dionex ICS-900) while dissolved Silica $\left(\mathrm{Si}(\mathrm{OH})_{4}\right)$, hereafter considered as $\mathrm{SiO}_{2}$, was determined by using an ICP-AES. The reliability of the chemical analyses was verified by using an ionic balance error (IBE) equation (Appelo and Postma, 2005). The values were within $\pm 5 \%$; hence, suitable for geochemical interpretations.

\section{RESULTS AND DISCUSSION}

\section{Physico-chemical composition of groundwater and surface water}

Table 1 presents results of the 72 water samples with a statistical summary shown in Table 2 . A statistical summary 
of rain data is presented in Table 3 . Mean depth to the water table $(<27 \mathrm{~m})$ and depth of the water column $(<8.4$ $\mathrm{m}$ ) were low, suggesting a shallow aquifer system. The mean temperature of the water was closer to the mean atmospheric temperature of $26^{\circ} \mathrm{C}$, suggesting present day climatic influence and recharge. Ground-water had a lower and narrow range in $\mathrm{pH}$ with a mean of 5.9, moderately acidic, while surface water had a relatively higher and wider range in $\mathrm{pH}$ with a mean of 6.8 , slightly acidic. About $88 \%$ of the 72 samples had $\mathrm{pH}<7$, suggesting an acidic aquifer system. The EC and TDS values of all water sources were low with mean values of $60 \mu \mathrm{S} / \mathrm{cm}$ and $39 \mathrm{mg} / \mathrm{l}$, respectively suggesting lowmineralised and freshwater. Major ion concentrations, like TDS, were low (Table 2) and in the order (meq/l) $\mathrm{Na}^{2+}>$ $\mathrm{Ca}^{2+}>\mathrm{Mg}^{2+}>\mathrm{K}^{+}$for cations and $\mathrm{HCO}_{3}^{-}>>\mathrm{NO}_{3} \geq \mathrm{Cl}^{-}>\mathrm{SO}_{4}{ }^{2-}$ for anions. The small range in their concentrations and higher dispersions around their respective mean values as reflected by the small STD values, except for $\mathrm{NO}_{3}{ }^{-}$and $\mathrm{Cl}^{-}$(Table 2), suggests a unique source of most ions and a single aquifer system. Mean concentration of $\mathrm{SiO}_{2}$ was higher than that of individual cations but less than the mean of $\mathrm{HCO}_{3}{ }^{-}$(Table 2). This suggests that $\mathrm{SiO}_{2}$ was released more than the cations into solution. The high $\mathrm{SiO}_{2}$ suggests the abundance of silicate minerals (Srinivasamoorthy et al., 2008) in agreement with the dominant granitic basement (Figure 1) and as observed in similar terrains by Njitchoua and Ngounou-Ngatcha (1997) and Fantong et al. (2009).

As expected, the mean TDS increased progressively from a minimum in rainwater $(20 \mathrm{mg} / \mathrm{l})$ to surface water (30 mg/l) and a maximum in groundwater $(44 \mathrm{mg} / \mathrm{l})$. Similarly, mean relative concentrations of dissolved ions showed a progressive increase from the lowest in rainwater, to surface water, and the highest in groundwater (Figure 2a). These results suggest chemical evolution from rain to surface water and groundwater. The relatively high TDS in groundwater indicated the influence of the unconsolidated sediments and granitic bedrock material in chemically enriching the water as it percolates through the under-saturated zone (or flows through the aquifer). The small difference in mean TDS of groundwater and rainwater by only $53 \%$ is likely because these are early rains, usually enriched in dissolved ions (Takounjou et al., 2011). The low major ion concentrations in groundwater depict low water-rock interactions in the granitic basement, short residence time, the shallow nature of the aquifer and its acidic nature (Edmunds and Smedley, 1996). Similar low $\mathrm{pH}$ and major ions have been reportedfrom granitic basement complex aquifers in Ghana (Adomako et al., 2011) and Nigeria (Edet et al., 2011).

\section{Hydrochemical facies}

The meq/l concentrations of major ions plotted on a pie chart (Figure 2b), showed that $\mathrm{Na}^{+}, \mathrm{HCO}_{3}{ }^{-}$and to some extent $\mathrm{Ca}^{2+}$ were the major ionic species. Low $\mathrm{K}^{+}$may be due to its low geochemical mobility (Hem, 1989; Srinivasamoorthy et al., 2008) while low $\mathrm{Mg}^{2+}$ is possibly due its to low content in rocks of the study area. On a Piper diagram (computed using GW_Chart Version 1.23.7.0), most samples plotted in the field of alkali metals $(\mathrm{Na}+\mathrm{K})$ (Figure 3$)$. This indicates the dominance of alkali metals in the groundwater aquifer system. The water types and their proportions are shown (Figure 3). The observed spatial distribution of the water types (Figure 1) showed no discernible pattern from $\mathrm{Ca}-\mathrm{HCO}_{3}$ to $\mathrm{Na}-\mathrm{HCO}_{3}$. Given the gentle relief in the plain, this suggests a mixed aquifer system as revealed by the mixed $\mathrm{Na}-\mathrm{Ca}-\mathrm{HCO}_{3}$ water. Similarly, no clear pattern, in water type, was observed from surface water to groundwater (Figure 3). Nonetheless, there is chemical evolution from $\mathrm{Ca}+\mathrm{Mg}+\mathrm{SO}_{4}+\mathrm{Cl}$ (rain), to $\mathrm{Ca}-\mathrm{HCO}_{3}$ (largely groundwater and lakes), and to $\mathrm{Na}-\mathrm{HCO}_{3}$ (basically groundwater, streams, rivers, and dam waters) as indicated by the dotted arrow (Figure 3). It is probable that the dissolution of $\mathrm{Na}^{+}$into surface waters from alkali-rich trachytic rocks in the surrounding highlands tends to mask the chemical evolution. The presence of $\mathrm{Ca}-\mathrm{HCO}_{3}$ water in groundwater within the plain, which is sporadic, in surface waters (streams and rivers) discharging from the highlands suggests that the main recharge of groundwater may not necessarily occur at higher altitudes but rather through direct precipitation to the surface and subsequent percolation through the under-saturated zone into the aquifer.

\section{Origin of solutes and hydrochemical controls}

The major ion chemistry of groundwater and compositional relations among ionic species can reveal the origin of solutes and processes that generated an observed water composition (Hounslow, 1995; Demlie et al., 2007; Cendon et al., 2011; Tay, 2012). Table 4 shows correlation coefficients between the ionic species in water. The good positive correlation of $\mathrm{NO}_{3}{ }^{-}$with $\mathrm{Cl}^{-}$suggests their anthropogenic origin (Demlie et al., 2007; Fantong et al., 2009). Main potential sources of $\mathrm{NO}_{3}{ }^{-}$are numerous shallow pit toilets and oxidation of organic matter given the proximity $(>15 \mathrm{~m})$ to shallow groundwater and agricultural dominance, respectively. Fonge et al. (2012) identified relatively high concentrations of $\mathrm{NO}_{3}{ }^{-}$ and $\mathrm{PO}_{4}{ }^{3-}$ in soil water $(<5 \mathrm{~cm}$ deep) from rice farms in the study area. The low content of $\mathrm{NO}_{3}{ }^{-}$and the absence of $\mathrm{PO}_{4}{ }^{3-}$ in groundwater suggest their sorption on organic colloids (in the under-saturated source of zone) or the location of rice fields rather in low-lying swampy areas. The likely $\mathrm{Cl}^{-}$is atmospheric inputs given the positive correlation with $\mathrm{Na}^{+}$and disinfection of wells through chlorination as noted in the field. Sulfur occurs in a reduced form in igneous rocks and is oxidized to $\mathrm{SO}_{4}{ }^{2-}$ when weathered in contact with aerated water (Hem, 1989). The significant positive correlations of $\mathrm{SO}_{4}{ }^{2-}$ with $\mathrm{K}^{+}, \mathrm{Ca}^{2+}$ and $\mathrm{HCO}_{3}^{-}$possibly suggest its igneous origin in 
Table 1. Physico-chemical results of groundwater and surface water $(n=72)$ in the Ndop plain (January-February, 2012).

\begin{tabular}{|c|c|c|c|c|c|c|c|c|c|c|c|c|c|c|c|}
\hline ID & $T$ & $\mathrm{pH}$ & EC & TDS & $\mathrm{Na}^{+}$ & $\mathrm{K}^{+}$ & $\mathrm{Ca}^{2+}$ & $\mathrm{Mg}^{2+}$ & $\mathrm{HCO}_{3}^{-}$ & $\mathrm{NO}_{3}^{-}$ & $\mathrm{Cl}^{-}$ & $\mathrm{SO}_{4}{ }^{2-}$ & $F^{-}$ & $\mathrm{SiO}_{2}$ & IB \\
\hline OW06 & 25.4 & 5.7 & 15 & 9.8 & 1.0 & 0.9 & 0.2 & 0.4 & 6.3 & 0.1 & 0.1 & 0 & 0.03 & 9.9 & 2 \\
\hline OW07 & 24.3 & 5.9 & 28 & 18.2 & 2.0 & 1.0 & 0.6 & 0.8 & 10.6 & 1.4 & 0.2 & 0.02 & 0.04 & 12.1 & 1 \\
\hline OW09 & 24.4 & 6.2 & 25 & 16.3 & 0.4 & 0.9 & 1.7 & 0.3 & 7.9 & 0.2 & 0.1 & 0.54 & 0.02 & 7.7 & 2 \\
\hline OW10 & 24.3 & 5.5 & 19 & 12.4 & 1.3 & 0.6 & 0.4 & 0.7 & 8.2 & 0.5 & 0.1 & 0 & 0.02 & 10.8 & 3 \\
\hline OW18 & 23.8 & 5.6 & 9 & 5.9 & 0.2 & 0.5 & 0.3 & 0.1 & 2.5 & 0.1 & 0.1 & 0 & 0.01 & 3.8 & 7 \\
\hline OW19 & 24.0 & 5.8 & 52 & 33.8 & 6.1 & 1.0 & 0.4 & 0.6 & 21.0 & 0.6 & 0.1 & 0.04 & 0.07 & 3.7 & 1 \\
\hline OW20 & 23.6 & 5.8 & 59 & 38.4 & 1.4 & 2.0 & 5.0 & 0.8 & 20.4 & 0.1 & 1.1 & 0.77 & 0.1 & 9.8 & 5 \\
\hline OW21 & 19.5 & 5.9 & 56 & 36.4 & 4.0 & 1.4 & 2.9 & 0.7 & 20.5 & 0.0 & 2.1 & 0.06 & 0.01 & 3.1 & 2 \\
\hline OW22 & 20.4 & 5.3 & 73 & 47.5 & 2.8 & 8.6 & 2.0 & 1.1 & 21.9 & 7.6 & 0.9 & 0.18 & 0.01 & 2.7 & 2 \\
\hline OW28 & 25.0 & 6.0 & 45 & 29.3 & 1.1 & 1.5 & 2.4 & 0.6 & 13.5 & 0.3 & 0.2 & 0.7 & 0.06 & 12.1 & 1 \\
\hline ow32 & 25.0 & 5.6 & 44 & 28.6 & 2.9 & 1.9 & 0.9 & 0.9 & 11.0 & 5.7 & 0.4 & 0.02 & 0.03 & 9.8 & 2 \\
\hline OW37 & 24.2 & 5.9 & 65 & 42.3 & 6.1 & 1.8 & 0.3 & 0.7 & 19.7 & 3.1 & 0.1 & 0.06 & 0.13 & 3.6 & 2 \\
\hline OW39 & 23.5 & 5.9 & 88 & 57.2 & 5.1 & 5.0 & 0.9 & 2.1 & 18.6 & 0.1 & 6.2 & 3.17 & 0.08 & 2.7 & 1 \\
\hline OW49 & 19.5 & 6.4 & 230 & 149.5 & 1.8 & 24.7 & 9.4 & 6.7 & 91.1 & 0.1 & 1.2 & 8.42 & 0.03 & 3.2 & 4 \\
\hline OW50 & 22.6 & 5.2 & 14 & 9.1 & 0.9 & 1.1 & 0.2 & 0.1 & 4.6 & 0.4 & 0.2 & 0.08 & 0.01 & 5.9 & 4 \\
\hline OW56 & 24.3 & 6.6 & 71 & 46.2 & 2.7 & 3.3 & 4.1 & 0.3 & 23.1 & 0.6 & 0.2 & 0.35 & 0.03 & 8.1 & 0 \\
\hline OW59 & 23.0 & 6.5 & 45 & 29.3 & 5.4 & 2.3 & 0.2 & 0.2 & 13.7 & 1.7 & 0.2 & 2.06 & 0.22 & 2.3 & 4 \\
\hline OW62 & 23.1 & 5.7 & 26 & 16.9 & 2.5 & 1.2 & 0.3 & 0.4 & 8.5 & 0.1 & 0.5 & 0.64 & 0.06 & 13.4 & 2 \\
\hline OW64 & 24.0 & 5.5 & 34 & 22.1 & 2.5 & 1.6 & 0.5 & 0.6 & 10.5 & 1.3 & 0.6 & 0.27 & 0.05 & 8.0 & 5 \\
\hline OW66 & 22.4 & 5.6 & 16 & 10.4 & 1.7 & 1.3 & 0.1 & 0.2 & 5.1 & 0.9 & 0.2 & 0.38 & 0.05 & 14.9 & 3 \\
\hline OW67 & 23.4 & 6.1 & 38 & 24.7 & 4.7 & 2.9 & 0.6 & 0.7 & 18.4 & 1.0 & 0.5 & 0.29 & 0.05 & 16.4 & 1 \\
\hline OW72 & 23.0 & 6.1 & 16 & 10.4 & 1.1 & 0.9 & 0.3 & 0.3 & 5.7 & 0.4 & 0.2 & 0.02 & 0.02 & 6.5 & 1 \\
\hline OW74 & 24.0 & 6.7 & 145 & 94.3 & 3.3 & 9.4 & 12.2 & 0.9 & 61.4 & 0.0 & 0.2 & 1.31 & 0.06 & 10.2 & 3 \\
\hline OW82 & 23.8 & 5.6 & 24 & 15.6 & 0.7 & 1.0 & 0.6 & 1.1 & 9.0 & 0.0 & 0.4 & 0.26 & 0.05 & 7.3 & 4 \\
\hline OW85 & 23.7 & 5.4 & 61 & 39.7 & 5.3 & 3.9 & 1.1 & 0.9 & 15.3 & 0.0 & 4.7 & 2.07 & 0.17 & 13.7 & 1 \\
\hline OW86 & 24.2 & 5.5 & 135 & 87.8 & 21.5 & 3.3 & 1.3 & 0.4 & 4.4 & 15.5 & 26.9 & 0.17 & 0.1 & 3.1 & 3 \\
\hline OW88 & 24.3 & 5.4 & 26 & 16.9 & 1.4 & 2.0 & 0.6 & 0.3 & 9.4 & 0.0 & 0.3 & 0.09 & 0.03 & 9.5 & 4 \\
\hline OW90 & 23.9 & 6.6 & 158 & 102.7 & 5.8 & 19.8 & 3.4 & 4.7 & 62.5 & 0.0 & 3.1 & 4.72 & 0.11 & 10.5 & 2 \\
\hline OW125 & 23.1 & 6.0 & 48 & 31.2 & 3.1 & 1.7 & 1.6 & 1.3 & 9.5 & 7.2 & 2.6 & 0.13 & 0.02 & 3.1 & 9 \\
\hline OW126 & 23.2 & 5.8 & 42 & 27.4 & 4.4 & 3.1 & 0.5 & 1.0 & 17.5 & 0.8 & 0.3 & 0.159 & 0.08 & 19.0 & 2 \\
\hline OW127 & 24.3 & 5.8 & 61 & 39.7 & 3.0 & 1.6 & 2.7 & 0.9 & 20.0 & 1.0 & 0.3 & 0.43 & 0.06 & 12.1 & 1 \\
\hline OW129 & 23.4 & 5.8 & 126 & 81.9 & 7.5 & 1.0 & 3.7 & 4.7 & 14.5 & 27.9 & 6.6 & 1.56 & 0.08 & 15.3 & 2 \\
\hline $\mathrm{BH} 02$ & 23.2 & 5.8 & 40 & 26.0 & 3.0 & 1.1 & 1.6 & 0.5 & 14.5 & 0.7 & 0.1 & 0.09 & 0.19 & 13.1 & 1 \\
\hline $\mathrm{BH} 11$ & 24.5 & 5.8 & 43 & 28.0 & 2.7 & 0.9 & 1.0 & 1.0 & 14.6 & 1.0 & 0.2 & 0.08 & 0.05 & 18.8 & 1 \\
\hline BH29 & 22.4 & 6.4 & 131 & 85.2 & 5.6 & 3.5 & 3.5 & 3.1 & 38.9 & 3.3 & 1.0 & 0.77 & 0.39 & 2.2 & 1 \\
\hline $\mathrm{BH} 41$ & 24.5 & 6.2 & 272 & 176.8 & 3.8 & 2.2 & 1.6 & 2.3 & 11.1 & 12.4 & 3.0 & 0.58 & 0.11 & 2.2 & 0 \\
\hline BH52 & 25.1 & 6.3 & 45 & 29.3 & 1.4 & 1.3 & 2.7 & 0.2 & 13.4 & 0.7 & 0.2 & 0.27 & 0.02 & 6.6 & 5 \\
\hline BH53 & 24.0 & 5.4 & 34 & 22.1 & 1.5 & 3.3 & 1.4 & 0.3 & 10.6 & 1.4 & 0.7 & 0.16 & 0.03 & 7.2 & 3 \\
\hline BH55 & 25.5 & 5.7 & 43 & 28.0 & 0.9 & 1.0 & 3.2 & 0.3 & 12.8 & 0.4 & 0.4 & 0.18 & 0.02 & 6.5 & 1 \\
\hline $\mathrm{BH} 60$ & 23.0 & 6.0 & 56 & 36.4 & 3.3 & 3.0 & 2.8 & 0.2 & 15.9 & 0.0 & 0.1 & 4.37 & 0.32 & 17.5 & -16 \\
\hline $\mathrm{BH} 70$ & 25.7 & 6.1 & 52 & 33.8 & 1.6 & 1.3 & 1.3 & 0.7 & 9.6 & 8.6 & 0.2 & 0.56 & 0.07 & 8.0 & 5 \\
\hline $\mathrm{BH} 73$ & 24.0 & 6.7 & 282 & 183.3 & 4.2 & 24.5 & 20.3 & 1.6 & 83.7 & 3.8 & 2.7 & 12.76 & 0.09 & 13.2 & 0 \\
\hline $\mathrm{BH} 76$ & 25.4 & 6.3 & 86 & 55.9 & 1.2 & 2.1 & 8.0 & 0.3 & 30.5 & 0.0 & 0.3 & 0.81 & 0.04 & 10.2 & 1 \\
\hline $\mathrm{BH} 77$ & 23.4 & 5.7 & 33 & 21.5 & 2.0 & 2.6 & 1.1 & 0.4 & 11.1 & 0.0 & 0.6 & 1.32 & 0.13 & 13.5 & 1 \\
\hline BH83 & 24.5 & 5.7 & 47 & 30.6 & 0.7 & 1.9 & 2.4 & 0.7 & 14.8 & 0.0 & 0.1 & 0.33 & 0.08 & 14.1 & 1 \\
\hline BH124 & 22.0 & 5.9 & 31 & 20.2 & 1.9 & 1.0 & 1.2 & 0.6 & 12.2 & 0.2 & 0.4 & 0.09 & 0.02 & 3.7 & 1 \\
\hline ST05 & 22.1 & 7.5 & 69 & 44.9 & 4.3 & 2.8 & 1.4 & 2.6 & 31.7 & 0.4 & 0.1 & 0 & 0.18 & 19.9 & 4 \\
\hline ST13 & 22.9 & 6.2 & 12 & 7.8 & 1.2 & 0.7 & 0.2 & 0.4 & 6.2 & 0.0 & 0.1 & 0 & 0.01 & 8.7 & 1 \\
\hline ST15 & 21.6 & 6.8 & 21 & 13.7 & 1.3 & 0.7 & 0.5 & 0.6 & 8.3 & 0.4 & 0.0 & 0 & 0.04 & 7.7 & 0 \\
\hline ST17 & 18.9 & 6.7 & 30 & 19.5 & 2.0 & 1.6 & 0.6 & 0.8 & 12.9 & 0.0 & 0.1 & 0 & 0.05 & 10.0 & 3 \\
\hline ST24 & 21.6 & 6.0 & 21 & 13.7 & 0.4 & 0.7 & 0.3 & 0.2 & 3.8 & 0.0 & 0.1 & 0.04 & 0 & 5.1 & 7 \\
\hline
\end{tabular}


Table 1. Contd.

\begin{tabular}{llllllllllllllll}
\hline ST27 & 20.1 & 5.8 & 19 & 12.4 & 1.2 & 0.7 & 0.3 & 0.3 & 5.0 & 0.0 & 0.3 & 0.06 & 0.07 & 5.8 & 1 \\
ST33 & 21.7 & 6.5 & 25 & 16.3 & 1.5 & 0.7 & 0.3 & 0.4 & 6.9 & 0.1 & 0.3 & 0.07 & 0.04 & 9.9 & 1 \\
ST40 & 20.9 & 7.2 & 110 & 71.5 & 5.5 & 2.0 & 0.8 & 4.7 & 41.6 & 0.1 & 0.3 & 0.03 & 0.18 & 2.7 & 1 \\
ST65 & 19.5 & 6.9 & 59 & 38.4 & 6.9 & 2.9 & 0.4 & 0.6 & 23.3 & 0.1 & 1.0 & 0.68 & 0.13 & 3.0 & 0 \\
ST68 & 19.4 & 6.9 & 44 & 28.6 & 4.7 & 2.0 & 0.3 & 0.7 & 19.1 & 0.1 & 0.2 & 0.16 & 0.08 & 19.7 & 1 \\
ST80 & 19.5 & 7.7 & 175 & 113.8 & 17.3 & 2.8 & 1.2 & 5.3 & 78.7 & 0.0 & 0.1 & 0.08 & 0.3 & 2.8 & 1 \\
ST87 & 21.1 & 6.4 & 58 & 37.7 & 7.2 & 3.1 & 0.2 & 0.3 & 24.4 & 0.0 & 0.3 & 0.06 & 0.06 & 2.8 & 2 \\
ST104 & 18.5 & 8.2 & 46 & 29.9 & 5.2 & 2.4 & 0.2 & 0.5 & 18.7 & 0.1 & 0.1 & 0.62 & 0.08 & 3.1 & 0 \\
ST108 & 19.2 & 7.9 & 29 & 18.9 & 4.3 & 2.6 & 0.1 & 0.3 & 14.4 & 0.2 & 0.2 & 0.47 & 0.1 & 3.0 & 3 \\
RW26 & 21.5 & 6.7 & 13 & 8.5 & 0.7 & 1.5 & 0.3 & 0.3 & 6.0 & 0.2 & 0.2 & 0.08 & 0.02 & 5.8 & 1 \\
RW42 & 20.1 & 6.7 & 80 & 52.0 & 4.8 & 2.1 & 0.8 & 2.7 & 29.5 & 0.6 & 0.4 & 0.26 & 0.09 & 2.3 & 1 \\
RW93 & 19.5 & 7.4 & 34 & 22.1 & 2.1 & 1.5 & 0.5 & 1.1 & 12.7 & 0.4 & 0.3 & 0.34 & 0.04 & 14.8 & 2 \\
RW94 & 21.0 & 7.1 & 39 & 25.4 & 4.0 & 1.9 & 0.2 & 0.3 & 14.2 & 0.1 & 0.2 & 0.35 & 0.08 & 20.5 & 1 \\
LK31 & 26.9 & 6.3 & 32 & 20.8 & 2.8 & 0.7 & 0.1 & 0.2 & 7.8 & 0.0 & 0.4 & 0.04 & 0.06 & 7.4 & 6 \\
LK43 & 21.7 & 8.4 & 33 & 21.5 & 1.2 & 1.0 & 1.8 & 0.2 & 8.8 & 0.0 & 0.6 & 0.56 & 0.03 & 11.6 & -1 \\
LK123 & 20.2 & 7.8 & 44 & 28.6 & 2.1 & 1.4 & 0.8 & 1.4 & 14.8 & 0.0 & 1.1 & 0.096 & 0.037 & 9.4 & 0 \\
DW16 & 25.3 & 6.5 & 47 & 30.6 & 3.7 & 2.6 & 0.4 & 0.9 & 16.5 & 0.0 & 0.9 & 0.01 & 0.07 & 15.9 & 3 \\
DW51 & 20.2 & 5.9 & 46 & 29.9 & 3.7 & 5.2 & 0.7 & 0.0 & 15.7 & 0.0 & 1.9 & 0.02 & 0.07 & 13.4 & 3 \\
DW54 & 22.7 & 5.5 & 24 & 15.6 & 2.9 & 2.0 & 0.4 & 0.8 & 14.4 & 0.0 & 0.3 & 0 & 0.06 & 12.2 & 4 \\
DW75 & 20.1 & 6.8 & 45 & 29.3 & 2.9 & 2.8 & 0.7 & 0.9 & 17.4 & 0.0 & 0.5 & 0 & 0.11 & 13.2 & 1 \\
DW128 & 27.3 & 5.8 & 58 & 37.7 & 1.7 & 0.9 & 1.0 & 1.1 & 12.9 & 16.1 & 0.8 & 0.413 & 0.03 & 9.1 & -35 \\
\hline
\end{tabular}

OW, Open well; BH, borehole; ST, stream water; RW, river water; LK, lake water; DW, dam water; EC, electrical conductivity ( $\mu$ S/cm); TDS, total dissolved solids (mg/l). All ions (mg/l); Br- was detected in only OW39 (0.02 mg/l); PO43-, in OW37 (0.43 mg/l), OW86 (0.1 mg/l) and LK43 (0.07 mg/l); NO2- $(>0.3 \mathrm{mg} / \mathrm{l})$ was detected in OW22, OW32, BH29, BH41 and LK16; IB, ionic balance.

Table 2. Statistical summary of physico-chemical data of groundwater and surface water in the study area.

\begin{tabular}{|c|c|c|c|c|c|c|c|c|c|c|c|c|}
\hline \multirow{2}{*}{ Parameter } & \multicolumn{4}{|c|}{ Surface and ground water $(n=72)$} & \multicolumn{4}{|c|}{ Groundwater $(n=46)$} & \multicolumn{4}{|c|}{ Surface water ( $n=26)$} \\
\hline & Min & Max & Mean & Std & Min & Max & Mean & STD & Min & Max & Mean & Std \\
\hline Alt. (m) & 1135 & 2272 & 1235 & 193 & 1163 & 1260 & 1188 & 19.24 & 1135 & 2272 & 1317 & 302 \\
\hline Dtw (m) & - & - & - & - & 1.60 & 27.00 & 8.81 & 5.06 & - & - & - & - \\
\hline Dow (m) & - & - & - & - & 0.30 & 8.40 & 1.57 & 1.36 & - & - & - & - \\
\hline $\operatorname{Tw}\left({ }^{\circ} \mathrm{C}\right)$ & 18.5 & 27.30 & 22.79 & 2.04 & 19.50 & 25.70 & 23.65 & 1.32 & 18.5 & 27.3 & 21.29 & 2.22 \\
\hline pH & 5.20 & 8.41 & 6.23 & 0.71 & 5.20 & 6.74 & 5.90 & 0.37 & 5.48 & 8.41 & 6.83 & 0.76 \\
\hline $\mathrm{EC}(\mu \mathrm{S} / \mathrm{cm})$ & 9.0 & 282 & 59.75 & 54.62 & 9.00 & 282 & 67.16 & 62.32 & 12.0 & 175 & 46.65 & 33.47 \\
\hline TDS (mg/l) & 5.85 & 183.30 & 38.84 & 35.5 & 5.85 & 183.3 & 43.65 & 40.51 & 7.8 & 113.75 & 30.33 & 21.76 \\
\hline $\mathrm{Na}^{+}(\mathrm{mg} / \mathrm{l})$ & 0.24 & 21.54 & 3.37 & 3.28 & 0.24 & 21.54 & 3.21 & 3.26 & 0.41 & 17.34 & 3.67 & 3.29 \\
\hline $\mathbf{K}^{+}(\mathrm{mg} / \mathrm{l})$ & 0.47 & 24.69 & 2.94 & 4.48 & 0.47 & 24.69 & 3.53 & 5.45 & 0.66 & 5.24 & 1.89 & 1.05 \\
\hline $\mathrm{Ca}^{2+}(\mathrm{mg} / \mathrm{l})$ & 0.11 & 20.25 & 1.80 & 3.02 & 0.11 & 20.25 & 2.50 & 3.58 & 0.14 & 1.77 & 0.56 & 0.41 \\
\hline $\mathrm{Mg}^{2+}(\mathrm{mg} / \mathrm{l})$ & 0.0 & 6.74 & 1.04 & 1.31 & 0.12 & 6.74 & 1.03 & 1.31 & 0.0 & 5.34 & 1.06 & 1.31 \\
\hline $\mathrm{HCO}_{3}^{-}(\mathrm{mg} / \mathrm{l})$ & 2.45 & 91.12 & 18.69 & 17.53 & 2.45 & 91.12 & 19.13 & 18.80 & 3.83 & 78.65 & 17.92 & 14.97 \\
\hline $\mathrm{NO}_{3}^{-}(\mathrm{mg} / \mathrm{l})$ & 0.0 & 27.92 & 1.80 & 4.51 & 0.00 & 27.92 & 2.41 & 5.05 & 0.00 & 16.07 & 0.73 & 3.07 \\
\hline $\mathrm{Cl}^{-}(\mathrm{mg} / \mathrm{l})$ & 0.04 & 26.87 & 1.13 & 3.31 & 0.05 & 26.87 & 1.53 & 4.07 & 0.04 & 1.88 & 0.42 & 0.42 \\
\hline $\mathrm{SO}_{4}{ }^{2-}(\mathrm{mg} / \mathrm{l})$ & 0.0 & 12.76 & 0.77 & 1.91 & 0.00 & 12.76 & 1.12 & 2.32 & 0.0 & 0.68 & 0.17 & 0.21 \\
\hline $\mathbf{F}^{-}(\mathrm{mg} / \mathrm{l})$ & 0.0 & 0.39 & 0.08 & 0.07 & 0.01 & 0.39 & 0.08 & 0.08 & 0.0 & 0.3 & 0.08 & 0.06 \\
\hline $\mathrm{SiO}_{2}(\mathrm{mg} / \mathrm{l})$ & 2.18 & 20.54 & 9.04 & 5.10 & 2.18 & 18.97 & 8.93 & 4.80 & 2.26 & 20.54 & 9.23 & 5.58 \\
\hline
\end{tabular}

Max, maximum, Min, minimum; STD, standard deviation; Alt, Altitude; Dtw, depth to water (m); Dow, depth of water (m); Tw, water temperature; EC, electrical conductivity; TDS, total dissolved solids.

agreement with the geology. Fluoride showed a distinct positive correlation with $\mathrm{Na}^{+}$relative to $\mathrm{Ca}^{2+}$ (Table 4).
The likely source of $\mathrm{F}^{-}$in the granitic basement is fluorite, $\mathrm{CaF}_{2}$, the most common $\mathrm{F}^{-}$-bearing mineral (Edmunds 
Table 3. Physico-chemical data of January 2012 sporadic rainfall events in the Ndop Plain and its Environs.

\begin{tabular}{lccccccccccccc}
\hline Location & $\mathbf{p H}$ & $\mathbf{E C}$ & $\mathbf{T D S}$ & $\mathbf{N a}^{+}$ & $\mathbf{K}^{+}$ & $\mathbf{C a}^{2+}$ & $\mathbf{M g}^{2+}$ & $\mathbf{H C O}_{3}{ }^{-}$ & $\mathbf{N O}_{3}{ }^{-}$ & $\mathbf{C l}^{-}$ & $\mathbf{S O}_{4}{ }^{2-}$ & $\mathbf{F}$ & $\mathbf{N a} / \mathbf{C a}$ \\
\hline Bamenda & 6.26 & 36 & 23.4 & 0.36 & 0.90 & 1.39 & 0.37 & 3.5 & 2.97 & 2.3 & 1.33 & 0.02 & 0.43 \\
Ndop & 6.57 & 30 & 19.5 & 0.26 & 0.88 & 1.62 & 0.43 & 3.77 & 2.58 & 1.56 & 1.25 & 0.03 & 0.27 \\
Ndawara & 6.45 & 28 & 18.2 & 0.29 & 0.87 & 1.30 & 0.36 & 3.97 & 2.47 & 1.43 & 1.12 & 0.01 & 0.37 \\
Kumbo & 6.53 & 32 & 20.8 & 0.23 & 1.09 & 1.37 & 0.27 & 4.12 & 3.73 & 1.22 & 2.03 & 0.03 & 0.28 \\
Max. & $\mathbf{6 . 5 7}$ & $\mathbf{3 6}$ & $\mathbf{2 3 . 4}$ & $\mathbf{0 . 3 6}$ & $\mathbf{1 . 0 9}$ & $\mathbf{1 . 6 2}$ & $\mathbf{0 . 4 3}$ & $\mathbf{4 . 1 2}$ & $\mathbf{3 . 7 3}$ & $\mathbf{2 . 3}$ & $\mathbf{2 . 0 3}$ & $\mathbf{0 . 0 3}$ & $\mathbf{0 . 4 3}$ \\
Min. & $\mathbf{6 . 2 6}$ & $\mathbf{2 8}$ & $\mathbf{1 8 . 2}$ & $\mathbf{0 . 2 3}$ & $\mathbf{0 . 8 7}$ & $\mathbf{1 . 3}$ & $\mathbf{0 . 2 7}$ & $\mathbf{3 . 5}$ & $\mathbf{2 . 4 7}$ & $\mathbf{1 . 2 2}$ & $\mathbf{1 . 1 2}$ & $\mathbf{0 . 0 1}$ & $\mathbf{0 . 2 7}$ \\
Mean & $\mathbf{6 . 4 5}$ & $\mathbf{3 1 . 5}$ & $\mathbf{2 0 . 5}$ & $\mathbf{0 . 2 9}$ & $\mathbf{0 . 9 4}$ & $\mathbf{1 . 4 2}$ & $\mathbf{0 . 3 6}$ & $\mathbf{3 . 8 4}$ & $\mathbf{2 . 9 4}$ & $\mathbf{1 . 6 3}$ & $\mathbf{1 . 4 3}$ & $\mathbf{0 . 0 2}$ & $\mathbf{0 . 3 4}$ \\
\hline
\end{tabular}

$\mathrm{EC}=\mathrm{uS} / \mathrm{cm}$, TDS and all ions $=\mathrm{mg} / \mathrm{l}, \mathrm{Na} / \mathrm{Ca}$

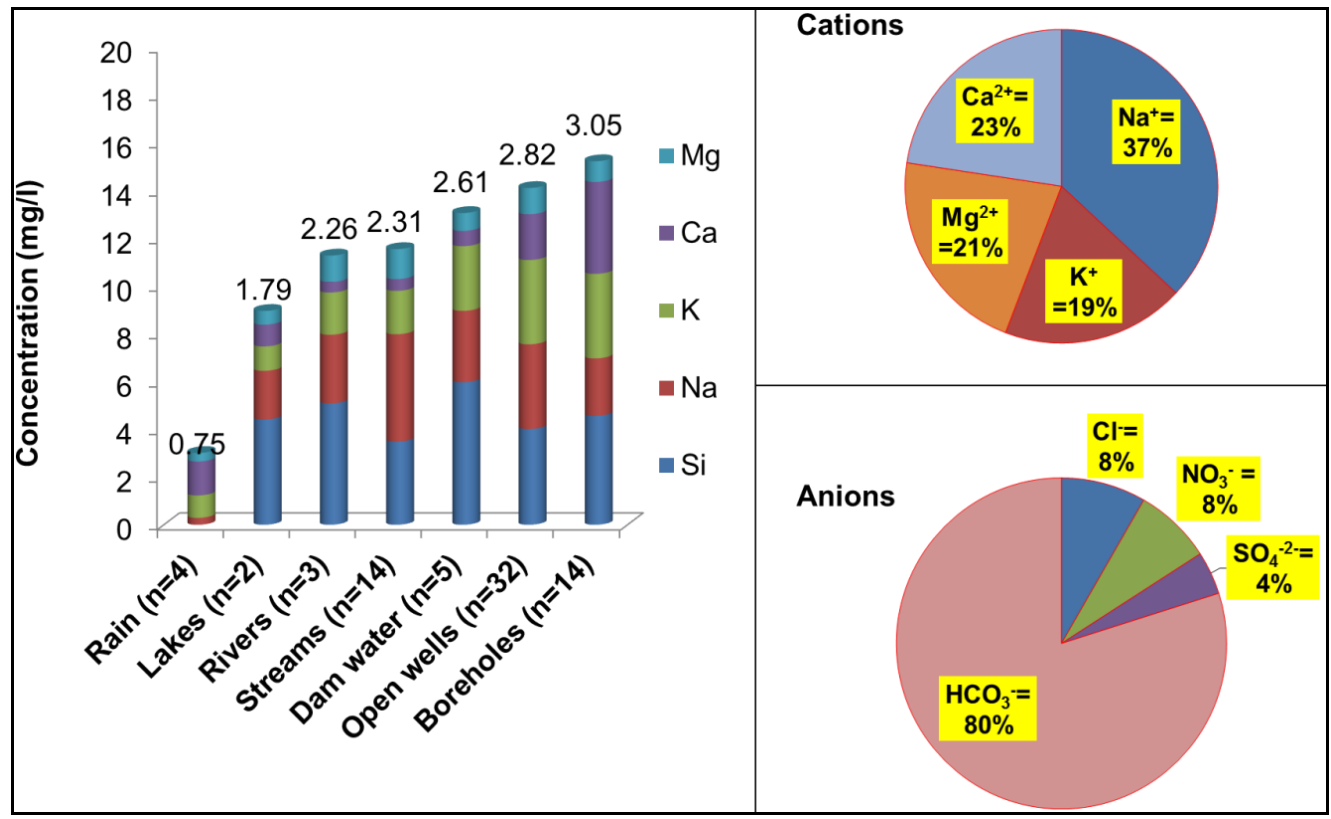

Figure 2. A, Relative mean concentrations of silica and cations $(\mathrm{mg} / \mathrm{l})$ in various water sources of the study area including rain. Si was not measured in rain; $\mathrm{Si}>\mathrm{Na}>\mathrm{K}>\mathrm{Ca}>\mathrm{Mg}$. B. Pie chart of mean concentrations of ions (meq/l), showing high $\mathrm{Na}+$ and dominant $\mathrm{HCO} 3-$ in water sources of the study area.

and Smedley, 1996). However, the released $\mathrm{Ca}^{2+}$ is possibly exchanged for $\mathrm{Na}^{+}$on clay surfaces, resulting in a relative increase in $\mathrm{F}^{-}$and a decrease in $\mathrm{Ca}^{2+}$, hence, the weak correlation between $\mathrm{Ca}^{2+}$ and $\mathrm{F}^{-}$.

High $\mathrm{F}^{-}$concentrations in groundwater have been identified in North Cameroon, along the CVL, in a graniticalkaline environment from fluorite, amphiboles and micas (Fantong et al., 2009). The low concentrations of $\mathrm{F}^{-}$in groundwater of the study area may be due to its acidic nature which renders $\mathrm{F}^{-}$immobile (Hem, 1989; Edmunds and Smedley, 1996). For example, the highest value of $\mathrm{F}^{-}$ $(0.39 \mathrm{mg} / \mathrm{l})$ was identified in $\mathrm{BH} 29$ ('borehole') with a $\mathrm{pH}$ of 6.4. The strong positive correlations of $\mathrm{HCO}_{3}{ }^{-}$with $\mathrm{Ca}^{2+}$, $\mathrm{Mg}^{2+}, \mathrm{K}^{+}$and $\mathrm{SO}_{4}{ }^{2-}$ further indicate similar origin of ions. Given the $\mathrm{Na}-\mathrm{HCO}_{3}$ dominance (Figure 3), the weak positive correlation of $\mathrm{Na}^{+}$with $\mathrm{HCO}_{3}{ }^{-}$suggests that varied processes accounts for its concentration in solution.

Table 5 shows a summary of computed ratios between some ionic species. The observed $\mathrm{Na}^{+} / \mathrm{Cl}^{-}$molar ratios were high, relative to that of sea water, 0.86 (Millero et al., 2008). $\mathrm{A} \mathrm{Na}^{+} / \mathrm{Cl}^{-}$molar ratio approximately equal to 1 is usually attributed to halite dissolution, whereas $>1$ is typically interpreted as reflecting $\mathrm{Na}^{+}$released from silicate weathering reactions (Fisher and Mullican, 1997; Cendon et al., 2010). Therefore, the excess $\mathrm{Na}^{+}$as further shown (Figure 4) is likely from silicate weathering, possibly feldspar mineral dissolution. On a plot of total cations, $\mathrm{Na}^{+}+\mathrm{K}^{+}+\mathrm{Ca}^{2+}+\mathrm{Mg}^{2+}(\mathrm{TC})$ versus alkalinity, most of the 72 samples (94\%) plotted on or near the 1:1 line (Figure 5a). The high mean $\mathrm{HCO}_{3} / 2$ anions ratio in the water (Table 5) and the 1:1 relationship (Figure 5a) suggest silicate or carbonate weathering (Hounslow, 1995; Fisher and Mullican, 1997; Kim, 2002), but acid waters (as prevailed in the study area) usually results from dissolution of non-carbonate rocks such as granites (Edmunds and Smedley, 1996), the dominant rock in the 


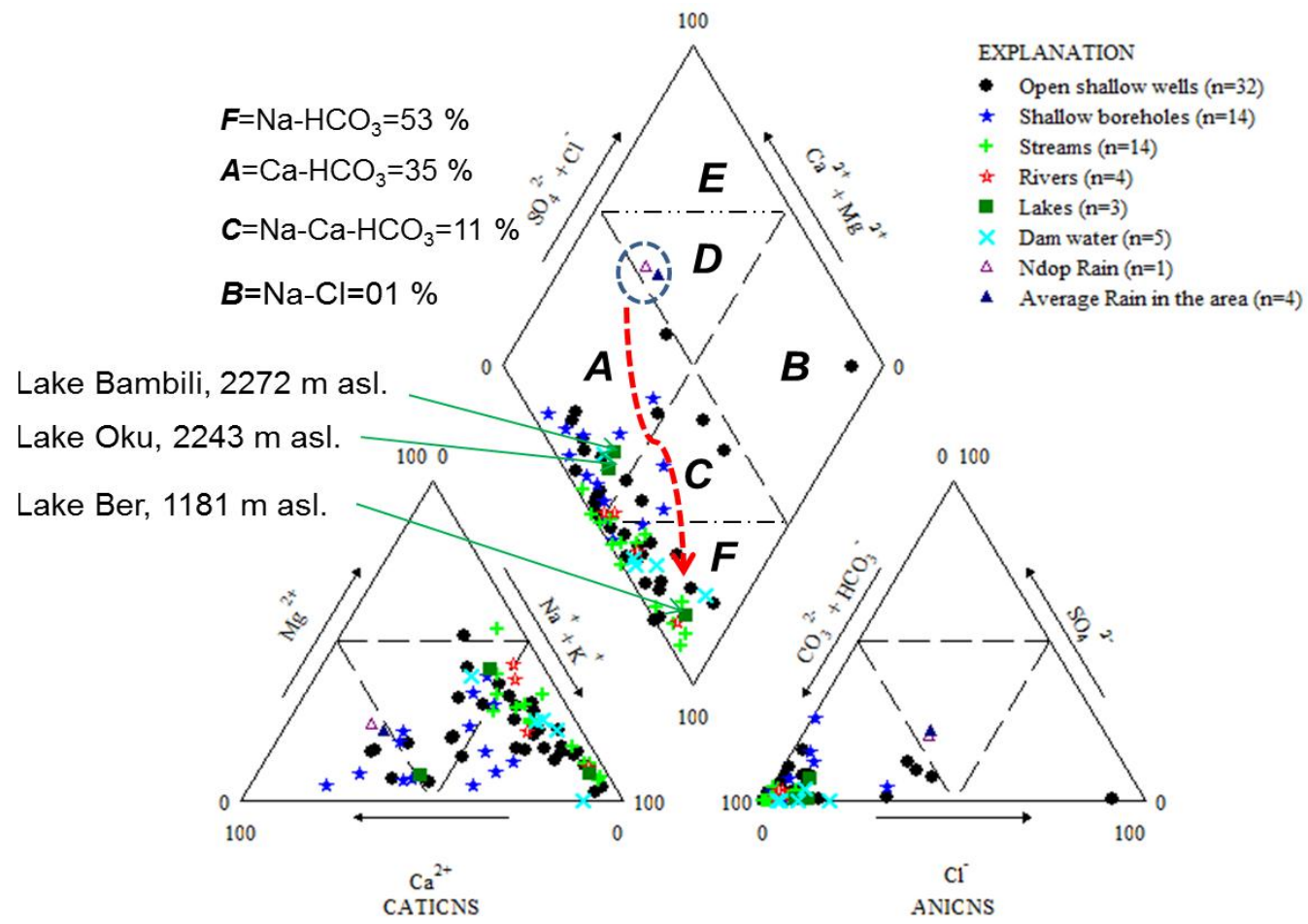

Figure 3. Piper's diagram showing the water types and proportions in the Ndop Plain. Alkali metals $(\mathrm{Na}+\mathrm{K})$ exceed alkali earth metals $(\mathrm{Ca}+\mathrm{Mg})$. Weak acids $\left(\mathrm{CO}_{3}+\mathrm{HCO}_{3}\right)$ greatly exceed strong acids $\left(\mathrm{SO}_{4}+\mathrm{Cl}\right)$. The dash line in the diamond section indicates the path of chemical evolution from $\mathrm{Ca}-\mathrm{Mg}$ $\mathrm{SO}_{4}-\mathrm{Cl}$ (rain), to $\mathrm{Ca}-\mathrm{HCO}_{3}$ (groundwater, lakes and dam water), and to $\mathrm{Na}-\mathrm{HCO}_{3}$ (groundwater, streams and rivers). $\mathrm{Ca}-\mathrm{Na}-\mathrm{HCO}_{3}$ represents a transition (mixing zone) from $\mathrm{Ca}-\mathrm{HCO} 3$ to $\mathrm{Na}-\mathrm{HCO} 3$ water.

Table 4. Pearson correlation coefficients of all analysed water sources $(n=72)$.

\begin{tabular}{|c|c|c|c|c|c|c|c|c|c|c|}
\hline Parameter & $\mathrm{Na}^{+}$ & $\mathrm{K}^{+}$ & $\mathrm{Mg}^{2+}$ & $\mathrm{Ca}^{2+}$ & $\mathrm{HCO}_{3}^{-}$ & $\mathrm{Cl}^{-}$ & $\mathrm{NO}_{3}^{-}$ & $\mathrm{SO}_{4}{ }^{2-}$ & $F^{-}$ & $\mathrm{pH}$ \\
\hline $\mathrm{Na}^{+}$ & 1.00 & & & & & & & & & \\
\hline $\mathrm{K}^{+}$ & 0.12 & 1.00 & & & & & & & & \\
\hline $\mathrm{Mg}^{2+}$ & 0.35 & 0.52 & 1.00 & & & & & & & \\
\hline $\mathrm{Ca}^{2+}$ & -0.01 & 0.73 & 0.27 & 1.00 & & & & & & \\
\hline $\mathrm{HCO}_{3}^{-}$ & 0.33 & 0.81 & 0.72 & 0.70 & 1.00 & & & & & \\
\hline $\mathrm{Cl}^{-}$ & 0.69 & 0.12 & 0.10 & 0.06 & -0.03 & 1.00 & & & & \\
\hline $\mathrm{NO}_{3}^{-}$ & 0.34 & -0.01 & 0.25 & 0.09 & -0.08 & 0.52 & 1.00 & & & \\
\hline $\mathrm{SO}_{4}{ }^{2-}$ & 0.05 & 0.87 & 0.40 & 0.77 & 0.68 & 0.11 & 0.04 & 1.00 & & \\
\hline$F^{-}$ & 0.48 & 0.07 & 0.36 & 0.04 & 0.35 & 0.07 & 0.01 & 0.17 & 1.00 & \\
\hline $\mathrm{pH}$ & 0.17 & 0.10 & 0.23 & 0.08 & 0.33 & -0.16 & -0.21 & 0.08 & 0.21 & 1.00 \\
\hline
\end{tabular}

area.

To further determine the likely influence of silicate dissolution, the molar ratio of $\mathrm{HCO}_{3}{ }^{-} / \mathrm{SiO}_{2}$, saturation indices for calcite and aragonite, and $\mathrm{Ca}^{2+}+\mathrm{Mg}^{2+}$ versus $\mathrm{SO}_{4}{ }^{2-}$ $+\mathrm{HCO}_{3}{ }^{-}$relationship were examined. If $\mathrm{HCO}_{3}{ }^{-} / \mathrm{SiO}_{2}$ ratio is greater than 10, (that is, $\mathrm{HCO}_{3}>>\mathrm{SiO}_{2}$ ), carbonate weathering predominates. However, if the ratio is low and especially $<5$, silicate weathering predominates (Hounslow, 1995). The computed $\mathrm{HCO}_{3}{ }^{-} / \mathrm{SiO}_{2}$ ratios (Table 5) were
$75 \%(<5), 19 \%(5-10)$ and $6 \%(>10)$. Calculated saturation indices (SI) of the water samples (using AqQA software) showed under-saturation $(S \mid<0)$ with respect to calcite and aragonite (Table 5) suggesting their absence in the formation and/or not enough time to interact. $A$ charge balance will exist between the $\mathrm{Ca}^{2+}+\mathrm{Mg}^{2+}$ and $\mathrm{SO}_{4}{ }^{2-}+\mathrm{HCO}_{3}^{-}$if they are derived from basic dissolution of calcite, dolomite, and gypsum (Fisher and Mullican, 1997). On the contrary, there was a deficiency in $\mathrm{Ca}^{2+}+\mathrm{Mg}^{2+}$ re- 
Table 5. A statistical summary of ratios between some ionic species in the analysed water.

\begin{tabular}{lcccc}
\hline Parameter & Minimum & Maximum & Mean & STD \\
\hline $\mathrm{Na}^{+} / \mathrm{Cl}^{-}$ & 1.25 & 5345 & $\mathbf{2 4 . 4 2}$ & 63.92 \\
$\mathrm{Na}^{+} / \mathrm{Ca}^{2+}$ & 0.13 & 27 & $\mathbf{5 . 3 5}$ & 6.66 \\
$\mathrm{HCO}_{3} /$ / anions & 0.06 & 68.34 & $\mathbf{9 . 6 0}$ & 10.93 \\
$\mathrm{HCO}_{3} / \mathrm{SiO}_{2}$ & 0.34 & 28.11 & $\mathbf{3 . 5 2}$ & 5.31 \\
$\mathrm{SI}$ of Calcite & -7.21 & -1.41 & $\mathbf{- 4 . 3 1}$ & 1.28 \\
$\mathrm{SI}$ of Aragonite & -7.38 & -0.17 & $\mathbf{- 4 . 3 9}$ & 1.43 \\
$\mathrm{R}_{\mathrm{E}}$ Index & 1.31 & 5.84 & $\mathbf{3 . 8 2}$ & 1.32 \\
PCO2 (atm) & 0.0000 & 0.1082 & $\mathbf{0 . 0 0 5 3}$ & 0.01 \\
Chloro-alkali index 1 (CAl 1) & -585 & -0.59 & $\mathbf{- 3 2 . 5 9}$ & 71 \\
Chloro-alkali index 2 (CAl 2) & -2.37 & -0.18 & $\mathbf{- 0 . 6 5}$ & 0.30 \\
Total Hardness & 0.97 & 57.27 & $\mathbf{8 . 7 7}$ & 10.48 \\
SAR & 0.08 & 4.34 & $\mathbf{0 . 6 4}$ & 0.65 \\
Kelly's Ratio & 0.08 & 10.03 & $\mathbf{1 . 7 0}$ & 2.12 \\
RSC (meq/I) & 0.07 & 2.52 & $\mathbf{0 . 4 8}$ & 0.48 \\
\hline
\end{tabular}

STD, Standard deviation; HCO3-/ $\Sigma$ anions (meq/l); SI, Saturation Index; RE (weathering index) $=2(3 \mathrm{~K}++3 \mathrm{Na}+2 \mathrm{Ca} 2+-$ $\mathrm{SiO} 2) / \mathrm{K}++\mathrm{Na}++\mathrm{Ca} 2+$ ), $\mathrm{RE}$ was calculated using molar concentrations of ions; PCO2 (Partial pressure of $\mathrm{CO} 2$ ) in water; $\mathrm{Na}+/ \mathrm{Ca} 2+$ $(\mathrm{meq} / \mathrm{l}) ; \mathrm{CAl} 1=\mathrm{Cl}-(\mathrm{Na}+\mathrm{K}) / \mathrm{Cl} ; \mathrm{CAl} 2=\mathrm{Cl}-(\mathrm{Na}+\mathrm{K}) /(\mathrm{SO} 4+\mathrm{HCO} 3+\mathrm{CO} 3+\mathrm{NO} 3)$. Total hardness $($ as $\mathrm{CaCO} 3 \mathrm{in} \mathrm{mg} / \mathrm{l})=2.5(\mathrm{Ca} 2+)+$ $4.1(\mathrm{Mg} 2+)$; SAR (Sodium Adsorption Ratio) $=\mathrm{Na}+/\{\mathrm{Ca} 2++\mathrm{Mg} 2+) / 2\} 0.5 ; \mathrm{KR}$ (Kelly's Ratio) $=\mathrm{Na}+/ \mathrm{Ca} 2++\mathrm{Mg} 2+$. RSC (Residual Sodium Carbonate $)=(\mathrm{CO} 32-+\mathrm{HCO}-)+(\mathrm{Ca} 2++\mathrm{Mg} 2) ; \mathrm{CAI}, \mathrm{SAR}, \mathrm{KR}, \mathrm{RSC}$ were calculated from ionic concentrations in meq/l.

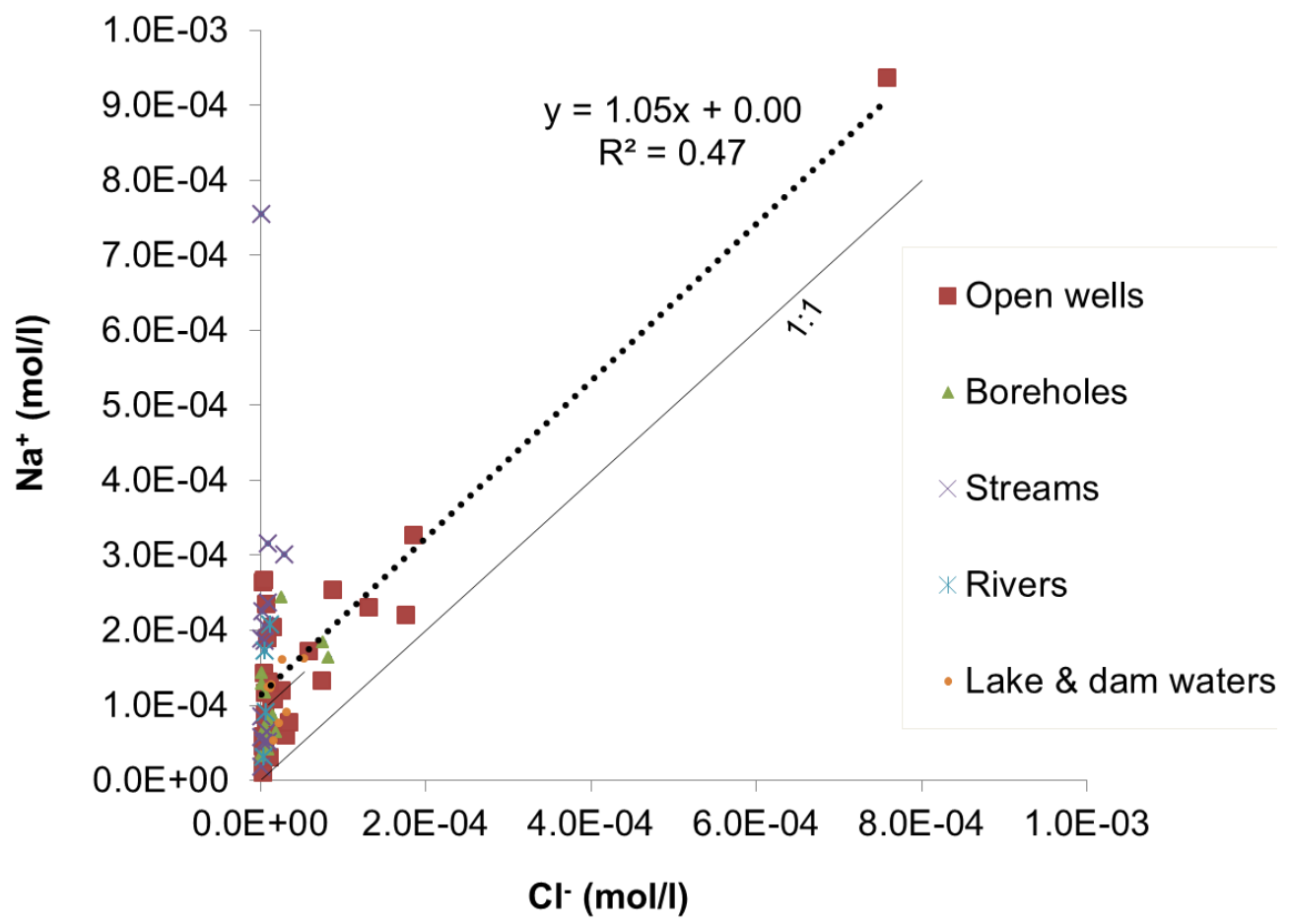

Figure 4. Plot of $\mathrm{Na}+$ as a function of $\mathrm{Cl}$ - showing an excess of $\mathrm{Na}+$ over $\mathrm{Cl}$ - in water sources.

lative to $\mathrm{SO}_{4}{ }^{2-}+\mathrm{HCO}_{3}{ }^{-}$(Figure $5 \mathrm{~b}$ ). Thus, the $75 \%$ dominance of samples with $\mathrm{HCO}_{3}{ }^{-} / \mathrm{SiO}_{2}$ ratios $<5$, calcite and aragonite under-saturation, and excess of $\mathrm{SO}_{4}{ }^{2-}+\mathrm{HCO}_{3}{ }^{-}$ relative $\mathrm{Ca}^{2+}+\mathrm{Mg}^{2+}$ strongly suggest incongruent weathering of silicate minerals (water-rock interaction) as the main control on water chemistry in agreement with the geology. Similar observations of silicate dissolution in granitic formations (Njitchoua and Ngounou-Ngatcha, 1997; Fantong et al., 2009) and volcanic terrains (Tanyileke et al., 1996; Ako et al., 2012) along the CVL have been reported with the following generalized equation (Tanyileke et al., 1996): 


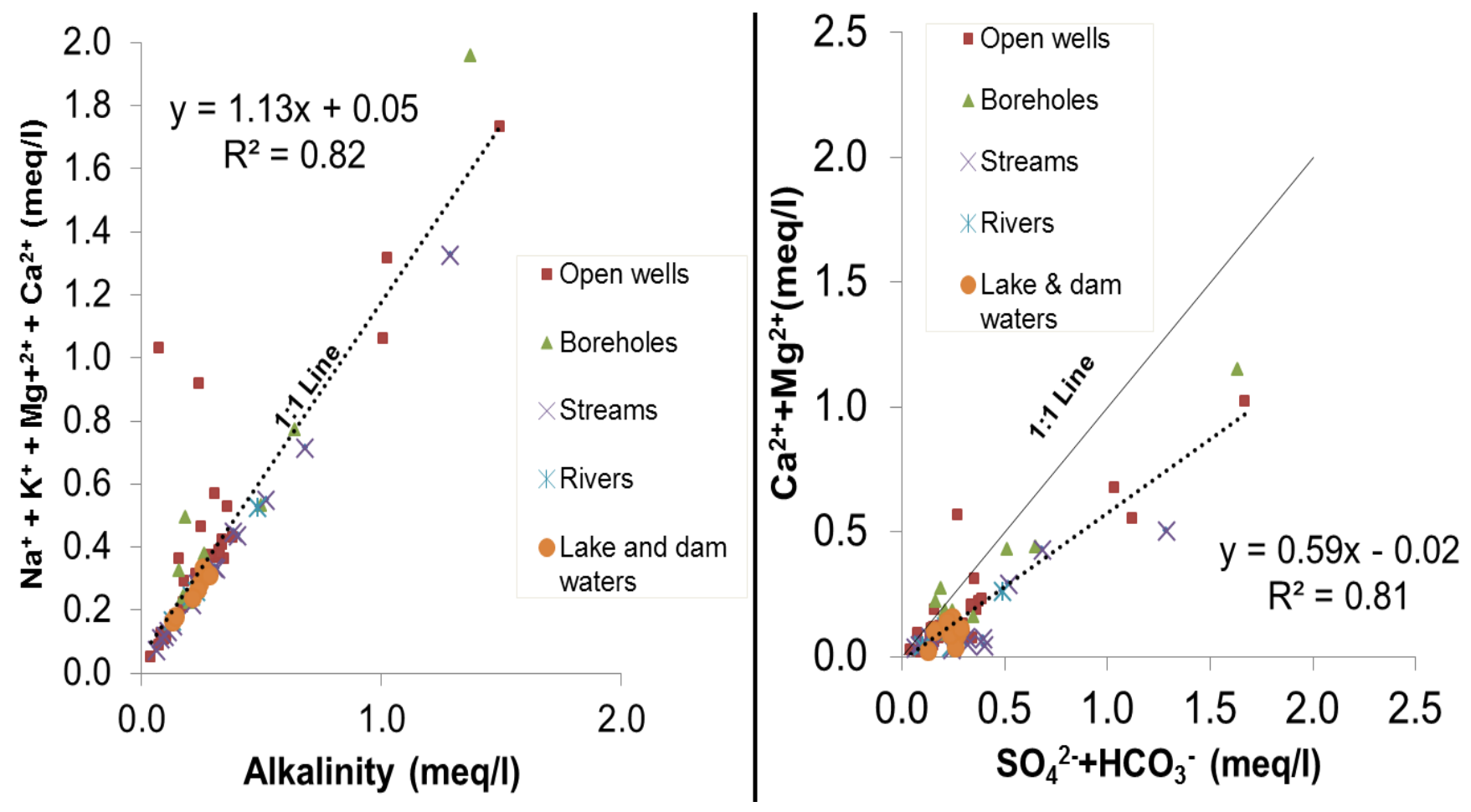

Figure 5. A, Plot of total cations as a function of alkalinity showing a 1:1 relationship in water sources of the study area. B, Plot showing deficiency in $\mathrm{Ca}^{2+}+\mathrm{Mg}^{2+}$ in relation to $\mathrm{SO}_{4}{ }^{2-}+\mathrm{HCO}_{3}^{-}$in water sources.

Rocks $+\mathrm{H}_{2} \mathrm{O}+\mathrm{CO}_{2}=$ Cations $+\mathrm{H}_{4} \mathrm{SiO}_{4}+\mathrm{HCO}_{3}{ }^{-}+$solids (mostly clay minerals).

The $\mathrm{HCO}_{3}{ }^{-}$dominance and high $\mathrm{SiO}_{2}$ are consistent with most natural waters along the CVL (Tanyileke et al., 1996).

Based on the geology and observed correlations between ionic species, the main mineral assemblage in the granitic basement under dissolution is possibly the plagioclase mineral series, with two end members, Albite $\left(\mathrm{NaAlSi}_{3} \mathrm{O}_{8}\right)$ and Anorthite $\left(\mathrm{CaAl}_{2} \mathrm{Si}_{2} \mathrm{O}_{8}\right)$. In the dissolution process, which involves the release of $\mathrm{SiO}_{2}$ and $\mathrm{HCO}_{3}{ }^{-}$ into solution and the formation of clay minerals (Equation 1), $\mathrm{Ca}^{2+}$ is preferentially released into solution more than $\mathrm{Na}^{+}$(Clayton, 1988; Deutsch, 1997). As expected, $\mathrm{Ca}^{2+}$ would be greater in groundwater, but as earlier observed, $\mathrm{Na}-\mathrm{HCO}_{3}$ dominated $\mathrm{Ca}-\mathrm{HCO}_{3}$ waters. Calculated Weathering Index $\left(R_{E}\right)$ using the formula of Tardy (1971) are shown in Table 5 . About $88 \%$ of samples had $\mathrm{R}_{E}$ values $<2$, while $12 \%$ had values $<2$. Accordingly, the weathering process in the study area resulted mainly in montmorillonite clay $\left(R_{E}>2\right)$ and minor kaolinite $\left(R_{E}>2\right)$ (Tardy, 1971). Montmorillonite has a high cation-exchange capacity (Appelo and Postma, 2005) and will probably result in ion exchange of $\mathrm{Ca}^{2+}$ in solution for $\mathrm{Na}^{+}$in rocks given the freshwater aquifer system (Hem, 1989) as des- cribed in this equation (Njitchoua and Ngounou-Ngatcha, 1997):

In order to determine the significance of base-exchange in enriching the water chemistry, a plot of $\mathrm{Ca}^{2+}+\mathrm{Mg}^{2+}$ $\mathrm{HCO}_{3}{ }^{-}-\mathrm{SO}_{4}{ }^{2-}$ versus $\mathrm{Na}^{+}-\mathrm{Cl}^{-}$was examined. If cation exchange is a significant groundwater composition-controlling process, the relation between these two parameters will be linear with a slope of -1.0 (Fisher and Mullican, 1997; Adomako et al., 2011; Tay, 2012). About $89 \%$ of the 72 samples plotted along the line with a slope of -0.99 (Figure $6 \mathrm{a}$ ). The 9 samples that diverted most from the line had relatively anomalous concentrations of cations or anions. A plot excluding these samples gave atrolling process, the relation between these two parameters will be linear with a slope of -1.0 (Fisher and Mullican, 1997; Adomako et al., 2011; Tay, 2012). About $89 \%$ of the 72 samples plotted along the line with a slope of -0.99 (Figure $6 \mathrm{a}$ ). The 9 samples that diverted most from the line had relatively anomalous concentrations of cations or anions. A plot excluding these samples gave a slope of -1.03 with an improved linearity (Figure 6b), indicating that ion exchange reactions occurred in a greater portion of the aquifer. The nature of ion exchange was determined by calculating Chloro-Alkaline Indices (CAI) (Schoeller, 1965). All samples gave negative values from CAI 1 and CAI 2 (Table 5). This indicated that $\mathrm{Mg}^{2+}$ 

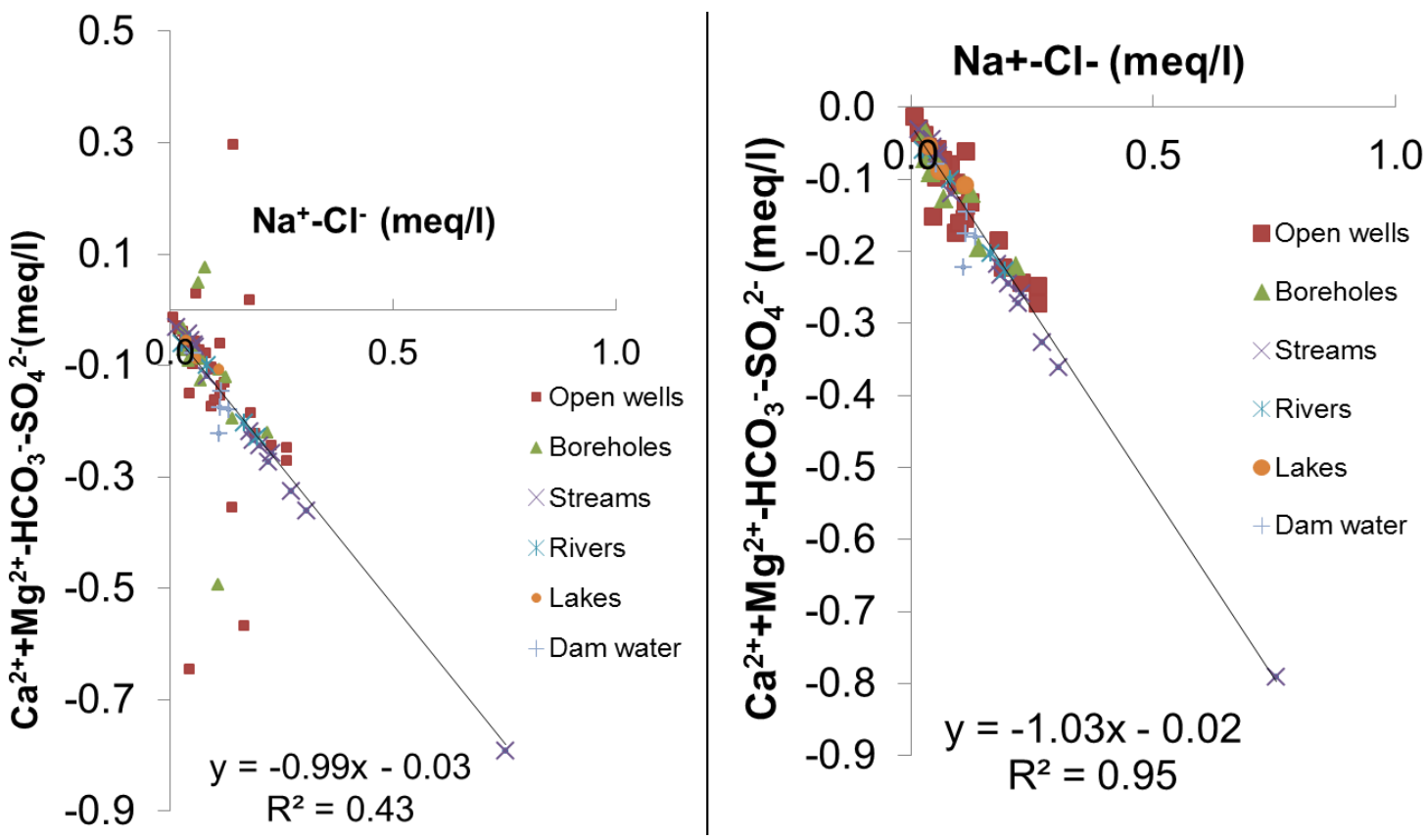

Figure 6. A, Relation of $\mathrm{Ca}^{2+}+\mathrm{Mg}^{2+}-\mathrm{HCO}_{3}+-\mathrm{SO}_{4}{ }^{2-}$ versus $\mathrm{Na}+-\mathrm{Cl}-$ showing an increase in $\mathrm{Na}+$ relative to a decrease in $\mathrm{Ca}^{2+}+\mathrm{Mg}^{2+}$ or an increase in $\mathrm{HCO}_{3}{ }^{-}$and $\mathrm{SO}_{4}{ }^{2-}$ with a slope close to -1.00 and a positive correlation $(n=72)$. B, Relation of $\mathrm{Ca}^{2+}+\mathrm{Mg}^{2+}-\mathrm{HCO}_{3}+-\mathrm{SO}_{4}{ }^{2-}$ versus $\mathrm{Na}+-\mathrm{Cl}$ - showing an increase in $\mathrm{Na}+$ relative to a decrease in $\mathrm{Ca}^{2+}+\mathrm{Mg}^{2+}$ or an increase in $\mathrm{HCO}_{3}{ }^{-}$and $\mathrm{SO}^{2-}$ with an improved linearity after excluding 9 anomalous samples $(n=63)$.

and $\mathrm{Ca}^{2+}$ in groundwater were being exchanged with $\mathrm{Na}^{+}$ and $\mathrm{K}^{+}$in rocks; hence, favouring cation-anion exchange reactions (chloro-alkaline disequilibrium) (Gupta et al., 2009). The $\mathrm{Na}^{+}$enrichment (addition) by ion exchange probably explains its observed weak positive correlation with other cations and $\mathrm{SO}_{4}{ }^{2-}$. Thus, besides the chemical dissolution of plagioclase feldspar minerals, cation-exchange reactions between water and montmorillonite in the aquifer also play a role in modifying the water composition.

The mean $\mathrm{P}_{\mathrm{CO} 2}$ in the groundwater (Table 5) was higher than the mean atmospheric value of $0.0003 \mathrm{~atm}$ (Appelo and Postma, 2005), suggesting that the major source of $\mathrm{CO}_{2}$ governing the above reactions was probably from the oxidation of organic matter in the undersaturated zone (Karanth, 1987; Appelo and Postma, 2005). The high $\mathrm{P}_{\mathrm{CO} 2}$ in groundwater are consistent with findings along the CVL (Njitchoua and Ngounou-Ngatcha, 1997; Fantong et al., 2009; Ako et al., 2012), which has been attributed to the same process. Unlike in these related studies, groundwater in the study is comparatively acidic. The acidic nature probably results from reduction in acid buffering capacity of infiltrating rainwater and leaching of organic constituents from soils into the shallow groundwater.

\section{Suitability for drinking and irrigation}

The drinking water guidelines of the WHO (2004) were used to evaluate the quality of water in the study area for human consumption. Most water sources had a cream colour, likely due to suspended organic colloids. Regardless of the cream colour, the range of all major ions and TDS were below the maximum values of the guidelines for drinking water. However, the $\mathrm{F}^{-}$content was low (0 to $0.39 \mathrm{mg} / \mathrm{l}$ ) with a mean of $0.08 \mathrm{mg} / \mathrm{l}$. At low concentrations $(<0.2 \mathrm{mg} / \mathrm{l})$ in drinking water, dental caries may result (Edmunds and Smedley, 1996). The water was soft, that is, low total hardness (Table 5) given the low $\mathrm{Ca}^{2+}$ and $\mathrm{Mg}^{2+}$. Their concentrations were mostly below the minimum required: $10 \mathrm{mg} / \mathrm{l}\left(\mathrm{Mg}^{2+}\right), 20 \mathrm{mg} / \mathrm{l}\left(\mathrm{Ca}^{2+}\right)$ and 60 $\mathrm{mg} / \mathrm{l}$ total hardness (Kozisek, 2005). Evidence is now available to confirm the existence of health risk from drinking water deficient in $\mathrm{Ca}^{2+}$ and $\mathrm{Mg}^{2+}$ (Rylander et al., 1991; Dissanayake and Chandrajith, 1999; Kozisek, 2005). Thus, the general water softness could be a health problem since soft water has been linked to cardiovascular ailments (Kozisek, 2005). About $69 \%$ of the water sources had $\mathrm{pH}$ values below 6.5. For effective disinfection of water with chlorine, the $\mathrm{pH}$ should preferably be less than 8 , but lower $\mathrm{pH}$ water $(<6.5)$ is likely to be corrosive $(\mathrm{WHO}$, 2004). High trace-metal contents, a health problem, are also likely from such low $\mathrm{pH}$ waters (Edmunds and Smedley, 1996), hence, a need to assess potentially harmful trace element. The low TDS in groundwater are consistent with the observation that most exploited groundwater resources in the weathered Precambrian basement rocks of Africa area low in dissolved ions and generally suitable for consumption (Adelana et al., 2011). However, the microbial quality of groundwater in the tropi- 
cal Africa is a serious health issue (Roche, 1993) as reported in the study area (Wirmvem et al., 2013) and elsewhere (Katte et al., 2003).

Suitability of water for irrigation has been assessed using various properties or ratios. These include EC, Sodium Adsorption Ratio (SAR), Kelley's Ration (KR) and Residual Sodium Carbonate (RSC) (Gupta et al., 2009; Ramesh and Elango, 2012). Electrical conductivity and SAR play a vital role in suitability of water for irrigation. The EC values in $99 \%$ of the analysed samples were $<250 \mu \mathrm{S} / \mathrm{cm}$ and SAR values were $<5$ (Table 5); thus, classifying the water into the low salinity hazard class (C1) and low sodium hazard class (S1), respectively (USSL, 1954; Gupta et al., 2009). Computed KR (Kelly, 1951) (Table 5) showed that $54 \%(K R<1), 25 \%(K R, 1-2)$, and $21 \%(K R>2)$ were suitable, marginal and unsuitable, respectively, for irrigation. Calculated RSC (Eaton, 1950) classified $93 \%$ and $7 \%$ of the water as suitable (RSC $\leq 1)$ and marginally suitable (RSC, 1.3-2.5), respectively, for irrigation.

\section{Conclusion}

A hydrochemical study, in the Ndop plain, to determine the physico-chemical characteristics, controls on water chemistry and suitability for drinking and irrigation has been carried out by analysing 72 water samples. The first physico-chemical baseline data of groundwater and surface water for water quality assessment, management and monitoring of changes in the hydrochemical system has been made available in the area. About $69 \%$ of the water sources had $\mathrm{pH}$ values below 6.5 , classifying the water as barely acidic. Therefore, careful attention is necessary at all stages of pipe borne water treatment and supply in the area as this could lead to corrosion and scale formation in pipes. The low mean EC $(60 \mu \mathrm{S} / \mathrm{cm})$ and TDS (39 mg/l) classify the water as low-mineralised and freshwater. Major ion concentrations were low, and below maximum values of the WHO standards for drinking water, but the low $\mathrm{Ca}^{2+}$ and $\mathrm{Mg}^{2+}$ classify the water as soft $\left(<<57 \mathrm{mg} / \mathrm{CaCO}_{3}\right)$. This could be a health problem given the relationship between soft water and cardiovascular ailments. The low $\mathrm{F}^{-}$content in the water sources $(<0.39 \mathrm{mg} / \mathrm{l})$ could lead to dental caries. In spite of these, the water sources are considered suitable for drinking as per WHO standards.

The relative abundance of major cations and anions in water $(\mathrm{meq} / \mathrm{ll})$ is $\mathrm{Na}^{+}>\mathrm{Ca}^{2+}>\mathrm{Mg}^{2+}>\mathrm{K}^{+}$and $\mathrm{HCO}^{-}>>\mathrm{NO}_{3}^{-}$ $\geq \mathrm{Cl}^{-}>\mathrm{SO}_{4}{ }^{2-}$, respectively. The main water types and proportions are $\mathrm{Na}-\mathrm{HCO}_{3}(53 \%), \mathrm{Ca}-\mathrm{HCO}_{3}(35 \%)$ and mixed $\mathrm{Na}-\mathrm{Ca}-\mathrm{HCO}_{3}(11 \%)$. Alkali metals $(\mathrm{Na}+\mathrm{K})$ exceed alkali earth metals $(\mathrm{Ca}+\mathrm{Mg})$ indicating their dominance in the aquifer system. The major controls on water chemistry are incongruent dissolution of silicate minerals (mainly plagioclase feldspars) and cation-exchange of $\mathrm{Na}^{+}$in rocks for $\mathrm{Ca}^{2+}$ in solution. The dissolution is governed by biogenic $\mathrm{CO}_{2}$ and results to montmorillonite clay, which enhances the ion exchange. The low $\mathrm{NO}_{3}{ }^{-}$and general absence $\mathrm{PO}_{4}{ }^{3-}$ in groundwater suggest their sorption by organic matter in the under-saturated zone. The low EC (TDS) and major ions in groundwater depict low solubility of the silicates, short residence time of groundwater, shallow nature of the aquifer and its acidic nature. The water sources are suitable for irrigation. Potentially harmful trace elements should be investigated given the acidic nature of the water. Hydrochemical investigations in the rainy season should also be carried out, to determine the seasonal controls on water chemistry.

\section{ACKNOWLEDGEMENTS}

This paper constitutes part of an on-going $\mathrm{PhD}$ research of the corresponding author, sponsored by the Japanese Government under the MONBUKAGAKUSHO Scholarship from the Ministry of Education, Culture, Sports, Science and Technology (MEXT). Material support is being provided by Japan Science and Technology Agency (JST) and Japan International Cooperation Agency (JICA) under the Science and Technology Research Partnership for Sustainable Development (SATREPS) project titled: Magmatic Fluid Supply into Lakes Nyos and Monoun, and Mitigation of Natural Disasters in Cameroon. The manuscript was improved by review comments from $\mathrm{Dr}$ Dion I. Cendon of Australian Nuclear Science and Technology Organization and an anonymous reviewer.

\section{REFERENCES}

Adelana S, Fantong W, Nedaw D, Duah A (2011). Groundwater and health: Meeting the unmet needs in Sub-Saharan Africa. In: Jones JAA (ed.) Sustainable groundwater resources. International Year of Planet Earth. Springer Science + Business Media B.V. pp. 21-33. DOI: 10.1007/978-90-481-3426-7_2.

Adelana SAM, MacDonald AM (2008). Groundwater research issues in Africa. In: Adelana SAM, MacDonald AM (eds.) Applied groundwater studies in Africa. Taylor and Francis, London. pp. 1-8.

Adomako D, Osae S, Akiti TT, Faye S, Maloszewski P (2011). Geochemical and isotopic studies of groundwater conditions in the Densu River Basin of Ghana. Environ. Earth Sci. 62:1071-1084.

Ako AA, Shimada J, Hosono T, Kagabu M, Akoachere RA, Nkeng GE, Eneke GT, Fouepe AT (2012). Spring water quality and usability in the Mount Cameroon area revealed by hydrogeochemistry. Environ. Geochem. Health 34 (5): 615-39.

Appelo CAJ, Postma D (2005). Geochemistry, groundwater, and pollution ( $2^{\text {nd }}$ ed.). Balkema, Amsterdam p. 635.

Cendon D I, Larsen JR, Jones BG, Nanson GC, Rickleman D, Hankin SI, Pueyo JJ, Maroulis J (2010). Freshwater recharge into a shallow saline groundwater system, Cooper Creek floodplain, Queensland, Australia. J. Hydrol. 392 (2-4): 150-163.

Clayton JL (1988). Some observations on the stoichiometry of feldspar hydrolysis in granitic soil. J. Environ. Qual. 17:153-157.

Demlie M, Wohnlich S, Wisotzky F, Gizaw B (2007). Groundwater recharge, flow and hydrogeochemical evolution in a complex volcanic aquifer system, central Ethiopia. Hydrogeol. J. 15: 1169-1181.

Deutsch WJ (1997). Groundwater geochemistry: Fundamentals and applications to contamination. CRC Press, Florida p. 221.

Dissanayake CB, Chandrajith R (1999). Medical geochemistry of tropical environments. Earth-Sci. Rev. 47: 219-258.

Eaton FM (1950). Significance of carbonates in irrigation waters. Soil Sci. 39: 123-133. 
Edet A, Nganje TN, Ukpong AL, Ekwere AS (2011). Groundwater chemistry and quality of Nigeria: A status review. Afr. J. Environ. Sci Technol. 5 (13): 1152-1169.

Edmunds WM, Smedley PL (1996). Groundwater geochemistry and health: An overview. In: Appleton JD, Fuge R, McCall GJH (eds.) Environmental geochemistry and health with special reference to developing countries. Geological Society Special Publication No.113, pp. 91-105.

Eneke GT, Ayonghe SN, Chandrasekharam D, Ntchancho R, Ako AA, Mouncherou OF, Thambidurai $P$ (2011). Controls on groundwater chemistry in a highly urbanised coastal area. Int. J. Environ. Res. 5 (2): 475-490.

Fantong WY, Satake H, Ayonghe SN, Aka FT, Asai K (2009). Hydrogeochemical controls and usability of groundwater in semi-arid Mayo Tsanaga River Basin, Far-North Cameroon. Environ. Geol. 58 (6): 1281-1293.

Fisher RS, Mullican WF (1997). Hydrochemical evolution of sodiumsulfate and sodium-chloride groundwater beneath the northern Chihuahuan Desert, Trans-Pecos, Texas, USA. Hydrogeol. J. 5: 416.

Fonge BA, Egbe EA, Fongod AN, Tening AS, Achu RM, Yinda GS, Mvondo ZEA (2012). Effects of land use on macrophyte communities and water quality in the Ndop wetland plain, Cameroon. J. Agric. Soc. Sci. 12: 41-49.

Gupta S, Dandele PS, Verma MB, Maithani PB (2009). Geochemical assessment of groundwater around Macherla-Karempudi area, Guntur District, Andhra Pradesh. J. Geol. Soc. India 73: 202-212.

Hem HD (1989). Study and interpretation of the chemical characteristics of natural waters $\left(3^{\text {rd }}\right.$ ed). U.S. Geological Survey Water-Supply Paper 2254.

Hounslow AW (1995). Water quality data: Analysis and interpretation. Lewis Publishers, Boca Raton.

Karanth KR (1987). Groundwater assessment, development and management. Tata McGrawl-Hill, New Delhi.

Karklins S (1996). Groundwater sampling desk reference. Wisconsin Department of Natural Resources Bureau of Drinking Water and Groundwater, PUBL-DG-037 96.

Katte VY, Fonteh MF, Guemuh GN (2003). Domestic water quality in urban centres in Cameroon: A case study of Dschang in the West Province. Afr. Water. J. Pilot ed.: 43-54

Kelly WP (1951). Alkali soils. Their formation, properties and reclamation. Reinhold Publication, New York.

Kim K (2002). Plagioclase weathering in the groundwater system of a sandy, silicate aquifer. Hydrol. Process. 16: 1793-1806.

Kozisek $F(2005)$. Health risks from drinking demineralised water. In: Nutrients in drinking water. World Health Organization, Geneva. pp. 148-163.

Marzoli A, Renne PR, Piccrilo EM, Francesca C, Bellieni G, Melfi A, Nyobe JB, N'ni J (1999). Silicic magmas from the continental Cameroon Volcanic Line (Oku, Bambouto and Ngaoundere): ${ }^{40} \mathrm{Ar}-{ }^{39} \mathrm{Ar}$ dates, petrology, Sr-Nd-O isotopes and their petrogenetic significance. Contrib. Mineral. Petr. 135: 133-150.

Millero FJ, Feistel R, Wright DG, McDougall TJ (2008). The standard composition of seawater: The Definition of a Reference Salinity Scale. Deep-Sea Res. I. 55 (1): 50-72.

Molua EL, Lambi CM (2006). Climate hydrology and water resources in Cameroon. CEEPA.

Ndenecho EN (2009). Cropping systems and post-cultivation vegetation succession: Agro- Ecosystem in Ndop Cameroon. J. Hum. Ecol. 27 (1): $27-33$
Ndzeidze SK (2008). Detecting changes in a wetland using multispectral and temporal Landsat in the Upper Noun Valley drainage basin-Cameroon. MSc. Thesis, Geography. Oregon State University.

Neba A (1999). Modern geography of the Republic of Cameroon ( ${ }^{\text {rd }}$ ed.). Neba Publishers, Bamenda p. 235.

Njitchoua R, Ngounou-Ngatcha B (1997). Hydrogeochemistry and environmental isotope investigations of the north Diamare plain northern Cameroon. J. Afr. Earth Sci. 25 (2): 307-316.

Ramesh K, Elango L (2012). Groundwater quality and its suitability for domestic and agricultural use in Tondiar river basin, Tamil Nadu, India. Environ. Monit. Assess. 184:3887-3899.

Roche MA (1993). Water quality Issues in the humid tropics. In: Bonell et al. (eds.) Hydrology and water management in the humid tropics: Hydrological research issues and strategies for water management. UNESCO. Cambridge University Press, Cambridge. pp. 344-366.

Rylander R, Bonevik H, Rubenowitz E (1991). Magnesium and calcium in drinking water and cardiovascular mortality. Scan. J. Work. Environ. Health 17 (2): 91-94.

Schoeller H (1965). Qualitative evaluation of ground water resources. In: Methods and techniques of groundwater investigation and development. Water Resourc. Series No. 33. UNESCO. pp. 44-52.

Srinivasamoorthy K, Chidambaram S, Prasanna MV, Vasanthavihar M, Peter J, Anandhan P (2008). Identification of major sources controlling groundwater chemistry from a hard rock terrain- A case study from Mettur taluk, Salem district, Tamil Nadu. J. Earth Syst. Sci. 117: 49-58.

Takounjou AF, Ngoupayou JRN, Riotte J, Takem GE, Mafany G, Marechal JC, Ekodeck GE (2011). Estimation of groundwater recharge of shallow aquifer on humid environment in Yaounde, Cameroon using hybrid water-fluctuation and hydrochemistry methods. Environ. Earth Sci. 64 (1): 107-118.

Tanyileke GZ, Kusakabe M, Evans WC (1996). Chemical and isotopic characteristics of fluids along the Cameroon Volcanic Line, Cameroon. J. Afr. Earth Sci. 22 (4): 433-441.

Tardy $Y$ (1971). Characterization of the principal weathering types by the geochemistry of waters from some European and African crystalline massifs. Chem. Geol. 7: 253-271.

Tay CK (2012). Hydrochemistry of groundwater in the Savelugu-Nanton District, Northern Ghana. Environ. Earth Sci. 67: 2077-2087.

US Salinity Laboratory Staff USSL (1954). Diagnosis and improvement of saline and alkali soils. Agricultural Handbook 60, USDA. US Government printing office, Washington DC.

Welz B, Becker-Ross H, Florek S, Heitmann U (2006). Front Matter, in High-Resolution Continuum Source AAS: The better way to do Atomic Absorption Spectrometry, Wiley-VCH Verlag $\mathrm{GmbH}$ \& Co. KGaA, Weinheim, FRG. Doi: 10.1002/3527606513.fmatter.

WHO (2004). Guidelines for drinking-water quality ( $3^{\text {rd }}$ ed.). Geneva, Switzerland.

WHO/UNICEF (2012). Joint monitoring programme for water supply and sanitation. Coverage estimates: Improved sanitation, Cameroon. www.wssinfo.org (Accessed: November 2012).

Wirmvem MJ, Fantong WY, Wotany ER, Ohba T, Ayonghe SN (2013). Sources of bacteriological contamination of shallow groundwater and health effects in the Ndop plain, Northwest Cameroon. J. Environ. Sci. Water Resourc. 2 (4): 127-132 